Les ANNALES Les Annales de droit

DE DROIT

$11 \mid 2017$

Varia

\title{
De « la constitution internationale »
}

Éléments de discussion sur les fondements de l'ordre juridique au-delà du cadre étatique

"The international constitution". Discussion of the foundations of the legal order beyond the State

\section{Rodrigue Ngando Sandjè}

\section{(2) OpenEdition}

Journals

Édition électronique

URL : http://journals.openedition.org/add/546

DOI : $10.4000 /$ add. 546

ISSN : 2606-1988

Éditeur

Presses universitaires de Rouen et du Havre

Édition imprimée

Date de publication : 16 octobre 2017

Pagination : 159-203

ISBN : 979-10-240-0775-5

ISSN : 1955-0855

Référence électronique

Rodrigue Ngando Sandjè, « De « la constitution internationale » », Les Annales de droit [En ligne],

11 | 2017, mis en ligne le 16 octobre 2018, consulté le 19 avril 2019. URL : http://

journals.openedition.org/add/546; DOI : 10.4000/add.546

Presses universitaires de Rouen et du Havre 


\title{
De «la constitution internationale »
}

\section{Éléments de discussion sur les fondements de l'ordre juridique au-delà du cadre étatique}

\author{
Rodrigue NGANDO SANDJE
}

\begin{abstract}
La raison d'être du petit État est qu'il $\mathrm{y}$ ait un endroit sur terre où le plus grand nombre possible de personnes puissent être des citoyens au plein sens du terme.
\end{abstract}

Jacob Burckhardt ${ }^{1}$.

Le syntagme «droit constitutionnel international » développé par le doyen Boris Mirkine-Guetzevitch porte empiriquement l'idée d'une constitution internationale ${ }^{2}$. On la situe sur deux plans (international et communautaire), mais toujours en rapport avec le cadre national ${ }^{3}$. Au plan international, la «constitution internationale», écrit Anne Peters, désigne «la somme des normes fondamentales qui réglementent les activités et relations politiques dans une politeia mondiale ${ }^{4}$ ». Dans cette perspective, la répartition des compétences au sein des organes de l'ONU

1. Cité par Oscar Leimgruber, «La Constitution de la Confédération Suisse de 1848 à 1948 », RID comp. 1949, vol. 1, n ${ }^{\text {os }} 1-2$, p. 22.

2. Voir Droit constitutionnel international, Paris, Sirey, 1933. Voir aussi Georges Scelle, «Le droit constitutionnel international» dans Mélanges offerts à Raymond Carré de Malberg, Paris, Librairie E. Duchemin, 1977, p. 501-515 ou encore Louis Favoreu, "Le contrôle de constitutionnalité du traité de Maastricht et le développement du "droit constitutionnel international" ", RGDI publ. 1993, n 1, p. 39-65.

3. Étienne Kenfack Temfack faisait remarquer le développement d'une «constitution internationale » placée au « frontispice» de la constitution nationale "pour lui définir son cadre et ses limites " (Le pouvoir constituant au Cameroun, thèse de droit public, Université de Douala, 2013, p. 578).

4. «Reconstruction constitutionnaliste du droit international: argument pour et contre », dans Hélène Ruiz Fabri et al. (dir.), Select Proceedings of the European Society of International Law, Oxford, Hart, 2008, vol. 1, p. 361. 
et entre celle-ci et les États ${ }^{5}$ correspondrait à la séparation des pouvoirs qui, dans le cadre national, est porteuse de "l'idée de constitution ${ }^{6}$ ». En cela, les auteurs comme Pierre-Marie Dupuy, considèrent le Conseil de sécurité des Nations unies comme le pouvoir exécutif de la communauté internationale ${ }^{7}$. Sur le plan régional européen, l'hypothèse d'une constitution apparait dans un double mouvement de constitutionnalisation du droit communautaire et de communautarisation du droit constitutionnel ${ }^{8}$. L'on explique pour une part que « les Constitutions des États membres codifient de plus en plus les principes communautaires: subsidiarité, cohésion économique et sociale, transfert aux institutions communautaires de compétences se rattachant à la souveraineté nationale » et, pour une autre part, que «le droit communautaire se constitutionnalise, à savoir il fait propre les principes fondamentaux incorporés dans les Constitutions des États membres: le principe démocratique, le principe de l'État de droit, les droits fondamentaux, la citoyenneté européenne ${ }^{9}$ ». En Afrique, l'évolution du régionalisme ${ }^{10}$ vers une « unité fédérative " promue au sein ${ }^{11}$ et au-delà ${ }^{12}$ des États entretient, elle aussi,

5. On pourrait indiquer à ce sujet le colloque puis l'ouvrage intitulé La charte des Nations unies, une constitution mondiale? (Paris, Pedone, 2006) présentés par Régis Chemain et Alain Pellet. Voir notamment les interventions de Jean-Marc Sorel («La répartition des compétences entre les organes des Nations unies») et d'Edwige Belliard ( La répartition des compétences entre l'ONU et les États »).

6. Voir l'article 16 de la DDHC. L'idée de constitution a fait l'objet d'une importante publication. Voir précisément Paul Bastid, L'idée de constitution, Paris, Economica, 1985.

7. Voir «The Constitutional Dimension of the Charter of the United Nations Revisited ", Max Planck Yearbook of United Nations Law, 2000, p. 21-25, disponible en ligne: http://www.mpil.de/files/pdf1/mpunyb_dupuy_12.pdf.

8. À un niveau plus global écrit Michel Bélanger, "[1]'internationalisation du droit constitutionnel favorise la constitutionnalisation du droit international» («Considérations sur la "constitutionnalisation internationale”: l'apport du droit international humanitaire", dans La constitution et les valeurs. Mélanges offerts à Dmitri Georges Lavroff, Paris, Dalloz, 2005, p. 44).

9. Sergio Marchisio, «Le droit communautaire dans les constitutions européennes», dans Rafâa Ben Achour et al. (dir.), Droit international et droits internes, développements récents, Paris, Pedone, 1998, p. 105-106. Jean Combacau conclurait à une « diminution de l'autonomie constitutionnelle des États membres des Communautés et de l'Union européennes» («Sources internationales et européennes du droit constitutionnel », dans Michel Troper et al. (dir.), Traité international de droit constitutionnel, Paris, Dalloz, 2012, t. I, p. 422).

10. François Borella, «Le régionalisme africain en 1964», AFDI 1964, p. 621-637. De manière générale voir Joseph-Marie Bipoun Woum, Le droit international africain: problèmes généraux, règlement des conflits, Paris, LGDJ, 1970.

11. Préambule de la Constitution du Burkina Faso.

12. Le Plan stratégique de la Commission de l'Union Africaine (2004), p. 25-26. 
la perspective d'une constitutionnalisation de l'ordre juridique supraétatique. Élisabeth Zoller ne dit-elle pas du fédéralisme qu'il est par excellence un processus international puisqu'il rapproche les peuples et les États ${ }^{13}$ ? La constitutionnalisation ainsi entretenue pourrait être envisagée à partir du rapport aux peuples, aux États ou encore au fédéralisme lui-même du moment où la constitution est consubstantielle autant à celui-ci ${ }^{14}$ qu'à ceux-là ${ }^{15}$. Il suit de ces nombreuses formulations l'idée d'un " constitutionnalisme multiniveaux » qui fait dire à Neil Walker que "les institutions, les normes et les pratiques constitutionnelles peuvent s'appliquer dans les contextes transcendant l'État ${ }^{16} »$. La question envisagée dans la présente étude n'est donc plus, pour reprendre des auteurs comme Michel Bélanger, du «domaine de la fiction ${ }^{17}$ ». Elle entend rendre compte des logiques postmodernes du droit marquées par la naissance d'une (ou de) nouvelle(s) constellation(s) politique(s) qui aiguise(nt) la curiosité ${ }^{18}$.

L'idée de «constitution internationale» entretient la problématique d'un ordre juridique au-delà du cadre national. Elle recèle plus d'un dilemme. Il y a celui toujours présent dans les esprits ${ }^{19}$ et qui oppose de manière beaucoup plus complexe aujourd'hui deux, voire trois, ordres

13. Voir "Aspects internationaux du droit constitutionnel. Contribution à la théorie de la fédération d'États », RCADI 2002, t. 294, p. 50.

14. Albert Venn Dicey, Introduction to the study of the Law of the Constitution, Londres, McMillan and $\mathrm{C}^{\circ}, 1902$, p. 140: "A federal state derives its existence from the Constitution, just as a corporation derives its existence from the grant by which it is created."

15. Élisabeth Zoller, Droit constitutionnel, Paris, PUF, 1999, p. 104.

16. Neil Walker, «Le constitutionnalisme multiniveaux» dans Michel Troper et al. (dir.), Traité international de droit constitutionnel, op. cit., t. I, p. 442.

17. "Considérations sur la "constitutionnalisation internationale"...», art. cité, p. 43. La première trace de l'idée de "constitution internationale" remonterait aux années 1920 suite aux travaux d'Alfred Verdross. Voir Die Einheit des rechtlichen weltbildes aufder Grundlage der Völkerrechtsverfassung, Wien, [s. n.],1923, p. 59 cité par Pascal Charlemagne Nyamnding Messanga, « Peut-on envisager la translation du concept de la constitution dans le cadre juridique international ? ", RCEI, $\mathrm{n}^{\circ}{ }_{1}$, 2007, p. 88-93.

18. Jürgen Habermas, Après l'État-nation: une nouvelle constellation politique, Paris, Fayard, 2000.

19. L'on pense au débat qui a opposé Hans Kelsen (Les rapports de système entre le droit interne et le droit international public, RCADI 1926) à Heinrich Triepel (Les rapports entre le droit interne et le droit international, RCADI 1923 ou encore Droit international et droit interne, R. Brunet (trad.), Paris, A. Pedone, 1920). 
juridiques autonomes, disons même indépendants ${ }^{20}$. Dans un avis $2 / 13$ rendu le 18 décembre 2014, la CJUE (assemblée plénière) rappelait à titre d'approximation l'autonomie - c'est le moins qu'on puisse dire de l'Union en tant que sujet de droit doté d'une personnalité juridique distincte des États qui la composent ${ }^{21}$. Puis, il y a l'exigence de qualification, en la supposant, de la notion de "constitution internationale» et, bien sûr, les implications d'une telle construction dans la théorie du droit.

L'enjeu consiste à rechercher l'aspect formel du fondement juridique au-delà de l'État puisqu'il a déjà été esquissé des points de repère qui pourraient concourir, par analogie, à la formulation de la dimension matérielle d'une "constitution internationale ${ }^{22}$ ».

Comment d'ailleurs méconnaître l'idée de constitution, si tant est qu'elle désigne «l'engendrement d'un objet et l'organisation de l'objet engendré ${ }^{3}$ ", dans un contexte dominé, exemple pris de l'Union européenne (UE), par un droit institutionnel ${ }^{24}$ qui poursuit, pour l'essentiel, la garantie des libertés et droits fondamentaux? La seule garantie des droits, au-delà du cadre étatique, ne justifierait-elle pas, en application de l'article 16 de la Déclaration des droits de l'homme et du citoyen (DDHC) du 26 août 1789 , l'hypothèse d'une "constitution internationale ${ }^{25} »$ ? Il conviendrait alors de comprendre la thèse de l'unité

20. «Le droit communautaire "dans son ensemble, constitue un système juridique nouveau, indépendant, supranational, autonome et unitaire, de caractère sui generis, avec un champ d'application limité" », disait Sadok Belaïd («Droit international et droit constitutionnel: les développements récents», dans Rafâa Ben Achour et al. (dir.), Droit international et droits internes, op. cit., p. 51).

21. Denys Simon, «Deuxième (ou second et dernier?) coup d'arrêt à l'adhésion de l'Union à la CEDH: étrange avis 2/13", Europe (Actualité du droit de l'union européenne), $\mathrm{n}^{\circ}$ 2, février 2015 , p. 8.

22. Pierre-Marie Dupuy, "The Constitutional Dimension of the Charter... », art. cité, p. 5-7.

23. Jean-Marie Denquin, «L'objet du droit constitutionnel: État, Constitution, démocratie », dans Michel Troper et al. (dir.), Traité international de droit constitutionnel, op. cit., t. I, p. 51.

24. L'on citera non exhaustivement Jacques Pertek, Droit des institutions de l'Union européenne, Paris, PUF, 2013 ; Jérôme Roux, Droit général de l'Union européenne, Paris, LexisNexis, 2012; Chahira Boutayeb, Droit institutionnel de l'Union européenne. Les institutions, l'ordre juridique, le contentieux, Paris, LGDJ, 2012 ou encore Droit institutionnel de l'Union européenne. La dynamique des pouvoirs, Paris, LGDJ, 2011 ; Joël Rideau, Droit institutionnel de l'union et des communautés européennes, Paris, LGDJ, 2002.

25. Pour certains auteurs, en effet, la garantie des droits marque l'effectivité de la constitution. Voir Vlad Constantinesco et al., Droit constitutionnel, Paris, PUF, 2011, p. 197. 
«constitutionnelle des peuples ${ }^{26}$ » développée dans ce terreau de la protection supra-étatique (communautaire et internationale) des droits et libertés comme concourant à la promotion d'une telle hypothèse.

Ainsi, comme l'aurait précisément écrit Pierre Brunet, le véritable enjeu réside dans la recherche des mécanismes d'énonciation du droit, notamment la définition du peuple auquel on imputerait la volonté générale ${ }^{27}$ qui sous-tend toute cette construction ${ }^{28}$, considérant le rapport peuple/constitution ${ }^{29}$ posé par le droit révolutionnaire ${ }^{30}$. Par ailleurs, il suivrait de l'idée de constitution l'unité entre le droit et l'État entretenue par des auteurs comme Hans Kelsen. La question sera celle de l'affirmation de l'État, corollairement à l'idée de constitution, au-delà du cadre national. Il se pourrait cependant que l'unité développée par Kelsen ne corresponde pas à une donnée naturelle ${ }^{31}$. Encore qu'à ce niveau, on pourrait ouvrir une parenthèse et relever qu'en application de l'article 16 de la DDHC, l'idée de constitution est liée à "toute société " et non exclusivement à l'État. L'unité développée par Kelsen se découvre donc dans un ensemble structuré de normes juridiques ${ }^{32}$ et bien plus encore, suivant la déontologie de l'école néoréaliste, dans la performance des acteurs du système de production de ces normes ${ }^{33}$. Dès lors, la définition matérielle de la "constitution internationale» ne consisterait pas ellemême à une caractérisation des règles et organes du système qu'ils fondent, mais à leur autorité à partir d'une logique concurrentielle entretenue par les différents acteurs interpellés par la dynamique du droit au sein de ce cadre ${ }^{34}$. Les multiples préoccupations qui se rapportent à

26. Jérôme Roux, Droit général de l'union européenne, op. cit., p. 25-90.

27. On sait en effet, depuis la décision 85-87 DC: Loi sur l'évolution de la NouvelleCalédonie, cons. 27, Rec. p. 70 du Conseil constitutionnel français, que la constitution est le déterminant de la volonté générale. Voir pour quelques développements, Philippe Blachèr, Contrôle de constitutionnalité et volonté générale, Paris, PUF, 2001.

28. Voir "Que reste-t-il de la volonté générale? Sur les nouvelles fictions du droit constitutionnel français ", Pouvoirs, n 114, 2005, p. 19.

29. Michel Troper, Pour une théorie juridique de l'État, Paris, PUF, 1994, p. 329.

30. Alexander Hamilton, Le fédéraliste. Lettre $n^{\circ} 78$ reproduit par David Mongoin, "Le fédéraliste revisité », Juspoliticum, $\mathrm{n}^{0} 8,2012$, http://juspoliticum.com/article/ Le-Federaliste-revisite-539.html.

31. Michel Troper, «Droit constitutionnel et théorie générale de l’État» dans Michel Troper et al. (dir.), Traité international de constitutionnel, op. cit., t. I, p. 209.

32. Pierre-Marie Dupuy, "The Constitutional Dimension of the Charter... », art. cité, p. 7-11.

33. Michel Troper, «Droit constitutionnel et théorie générale de l'État», art. cité, p. 201.

34. Hélène Tourard disait à ce propos que «l'internationalisation met en jeu un conflit de normes et donc un conflit de légitimité entre les autorités édictant ces normes ». Voir L'internationalisation des constitutions nationales, Paris, LGDJ, 2000, p. 167. 
l'idée d'une "constitution internationale» ne se posent donc pas faute d'éléments de réponse. Les hésitations observées s'expliqueraient par le poids du constitutionnalisme des Lumières, les choix méthodologiques ou le confort idéologique. Certains auteurs relèvent les difficultés à se départir de la théorie générale du droit ${ }^{35}$. D’autres concluent que la "Constitution fait partie des notions faussement claires de la science juridique $^{36} »$. Olivier de Frouville explique que «lorsqu'on se pose la question de l'existence d'une Constitution internationale, le problème n'est pas de savoir s'il existe, au plan international, quelque chose qui ressemblerait à la Constitution française de 1958 ; la question est plutôt de savoir si le concept de Constitution est inhérent au concept de Droit ${ }^{37}$ ». Peut-on, se demandait l'auteur, concevoir un « ordre juridique sans constitution ${ }^{38}$ ?».

Comment en effet nommer l'activité supranationale, voire transversale, de la production de normes qui interpelle les États et leurs citoyens? La notion de «citoyenneté mondiale ${ }^{39}$ " de plus en plus évoquée ne recèle-t-elle pas, pour autre approximation, l'idée d'une constitution? Toutes ces interrogations abondamment traitées auraient été surplombées par le culte du formalisme qui fait dire à Francis Hamon et Michel Troper que le privilège est donné au "point de vue formel, parce que c'est celui qui est adopté par la pratique juridique ${ }^{40} »$. Pour autant, le primat du critère formel ne devrait pas emporter l'idée de constitution sans inventaire. Marie-Anne Cohendet explique dans ce sens que «le critère formel devrait suffire à qualifier la constitution» dans le seul sens où il reflète la dimension fondamentale de la société que celle-là (la constitution) entend organiser. Sous-entendu que, «la forme n'a de raison d'être que de garantir la primauté et la stabilité du contenu»; à savoir « les principes essentiels de la société que le souverain veut établir, comme l'obligation de respecter les droits de l'homme, et d'autre part

35. Pierre Brunet, «La représentation» dans Michel Troper et al. (dir.), Traité international de droit constitutionnel, op. cit., t. I, p. 639.

36. Constance Grewe et al., Droits constitutionnels européens, Paris, PUF, 1995, p. 33.

37. Olivier de Frouville, «Paradigme de la constitutionnalisation vu du droit international », dans Stéphanie Hennette-Vauchez et al. (dir.), Les Droits de l'homme ont-ils constitutionnalisé le monde?, Bruxelles, Bruylant, 2011, p. 195.

38. Olivier de Frouville, «Paradigme de la constitutionnalisation vu du droit international », art. cité, p. 195.

39. Mireille Delmas-Marty, "Tribunaux internationaux et mondialisation", dans Emanuela Fronza et al. (dir.), La justice internationale dans les décisions des tribunaux ad hoc. Études des Law Clinics, Paris, Dalloz, 2003, p. 272.

40. Voir Droit constitutionnel, Paris, LGDJ, 2012, p. 34. 
les règles relatives à la production de normes ${ }^{41}$ ». Or il apparaît que la garantie des droits et libertés forme, avec les principes de démocratie et de sécurité, un principe essentiel de l'ordre juridique extranational ${ }^{42}$. L'idée d'une "constitution internationale " qui en suivrait correspondrait, suivant le mot de Paolo Comanducci, au modèle axiologique de la constitution conçue comme ordre ${ }^{43}$. D'évidence, la garantie visée impose des règles de production de normes aux niveaux national et supranational. Dès lors, il ne serait pas superflu de se pencher sur quelques analyses qui contribueraient à l'examen des fondements de l'ordre juridique supranational ${ }^{44}$, à la pratique juridique intégrée qu'on observe à grand relief en Europe et, en perspective, ailleurs ${ }^{45}$. Ne dit-on pas que le fait « [q]u'un phénomène soit ou non constitutionnalisé relève du fait et ne peut donner matière qu'à énumération ${ }^{46} »$ ?

Le travail pourrait s'avérer aisé puisque c'est sans conteste certains aspects - à partir de la pratique institutionnelle de la promotion des droits fondamentaux par exemple - de la « constitution internationale ${ }^{47} »$ qui ont déjà été développés. Mais les débats demeurent. S'il est en effet établi que «l'UE possède déjà clairement les caractéristiques constitutionnelles holistiques", peut-on étendre ces caractéristiques «à tous les niveaux de la pratique constitutionnelle moderne ${ }^{48}$ " ? Le moins que l'on puisse dire est que la question de la définition de la constitution au-delà du cadre étatique garde le mérite d'une permanente mise en perspective

41. Voir Droit constitutionnel, Paris, Montchrestien, 2002, p. 70-72.

42. Michel Bélanger, "Considérations sur la "constitutionnalisation internationale"...", art. cité., p. 44 et suiv.

43. Voir «Épistémologie» dans Michel Troper et al. (dir), Traité international de droit constitutionnel, op. cit., t. I, p. 19.

44. Neil Walker a pour sa part prédit le retour à la question de la constitution en Europe après la débâcle orchestrée par les référendums français et néerlandais de 2005. Voir «Le constitutionnalisme multiniveaux», art. cité, p. 455.

45. André Cabanis et al., "Un espace d'isomorphisme constitutionnel. L'Afrique francophone ", dans La constitution et les valeurs, op. cit., p. 343-358.

46. Jean-Marie Denquin, «L'objet du droit constitutionnel : État, Constitution, démocratie», art. cité, p. 63.

47. On parle d'un ordre juridique indépendant, complet, doté de règles lui permettant de se produire, de s'organiser, de s'étendre, de s'interpréter, de se corriger et de se discipliner lui-même. Voir Neil Walker, "Le constitutionnalisme multiniveaux », art. cité, p. 452.

48. Ibid., p. 455. 
du renouvellement épistémologique requis dans le domaine du droit ${ }^{49}$. Nous la suivrons à partir du double sens orthodoxe de la constitution, afin d'en relever les points significatifs, ceux qui regroupent la doctrine ou éloignent les écoles. On organisera schématiquement les questions soulevées à partir, d'une part, du sens formel de la constitution (1) et, d'autre part, de son sens substantiel (2).

\section{1. Éléments d'une catégorisation formelle de la «constitution internationale»}

Une kyrielle d'éléments désigne la constitution au sens formel: « un écrit unique à portée systématique (code) [mais aussi] un ensemble ou réseau de textes articulés autour d'un texte central à portée systématique ("galaxie")», une procédure singulière qui justifie les principes de rigidité et de supra-légalité ${ }^{50}$ ou encore l'auteur du texte: "un organe spécifique $^{51}$ » juridiquement indéterminé au moment de l'élaboration de la première constitution, mais dont on dit détenir le pouvoir du souverain originaire, le peuple ${ }^{52}$. De cette liste, non exhaustive, on pourrait tirer trois principes fédérateurs: la forme de la constitution (écrit), l'organe (pouvoir spécifique) et la procédure (spéciale, qui déroge à la procédure législative ordinaire).

Pour conventionnels qu'ils soient, les trois principes ne sont pas moins objets de curiosité. La forme (écrite) "prétendument» héritée du droit révolutionnaire ${ }^{53}$ ne fait par exemple pas défaut au-delà du cadre national. La parade - peu convaincante et du reste sans

49. Wagdi Sabète parle du grand questionnement de la science du droit, de l'évolution du système constitutionnel («Quelle théorie de la science juridique? Réflexion sur la représentation scientifique du droit public », $\left.\mathrm{RDP} 2000, \mathrm{n}^{\circ} 5, \mathrm{p} .1326\right)$. Christophe Jamin observe quant à lui l'effondrement des cathédrales doctrinales classiques ( «L construction de la pensée juridique française», dans Denys de Béchillon et al. (coord.), L'architecture du droit. Mélanges offerts à Michel Troper, Paris, Economica, 2006, p. 501).

50. Luc Heuschling, «La Constitution formelle », dans Michel Troper et al. (dir.), Traité international de droit constitutionnel, op. cit., t. I, p. 266.

51. Pierre Pactet et al., Droit constitutionnel, Paris, Sirey, 2012, p. 59.

52. Véronique Champeil-Desplats, «Théorie générale des sources du droit constitutionnel» dans Michel Troper et al. (dir.), Traité international de droit constitutionnel, op. cit., t. I, p. 236.

53. Rien en effet n'indique à la lecture de l'article 16 de la DDHC que l'idée de constitution renvoie à un texte écrit. 
grande importance ${ }^{54}$ - consiste à y ajouter la dénomination constitution puisque la notion de traité, contestée pour la cause, est présentée en tant que telle ${ }^{55}$. Carl Schmitt tranchait clairement en indiquant qu'un traité international «n'est jamais une constitution au sens positif ${ }^{56}$ ». La perspective est donc celle d'une "conception documentaire de la constitution ${ }^{57}$ ». Dans ce cas, la clause de «l'unanimité des États» ne saurait satisfaire l'exigence d'une procédure particulière. Une telle exigence aurait le défaut de disqualifier, à l'ordre étatique, l'idée de constitution chaque fois que celle-ci serait adoptée par une procédure ordinaire. C'est dans ce sens que Marie-Anne Cohendet soutient qu'une " “constitution souple" n'est pas formellement une constitution ${ }^{58}$ ". Des auteurs comme Jean-Marie Denquin réfutent dès lors l'élément procédural dans la définition de la constitution ${ }^{59}$. Le dernier élément, un organe spécifique, pose la question d'un peuple (le souverain) et du pouvoir constituant qui lui est consubstantiel. Il est le plus évoqué parce que représentatif de la philosophie révolutionnaire: «Rechercher le souverain ou le pouvoir constituant originaire est l'un des premiers réflexes du constitutionnalisme moderne », écrit dans ce sens Véronique Champeil-Desplats ${ }^{60}$. Ce critère permettra de poser le débat de la dimension politique (ou démotique en référence au peuple souverain) de l'ordre juridique supra-étatique ${ }^{61}$ (1.1). Après quoi, nous tirerons les leçons des positions adoptées en proposant, au dos du sacré, ce que pourrait vouloir dire une «constitution internationale» au sens formel du terme (1.2).

\subsection{La dimension démotique de la «constitution internationale» ou la recherche de l'éthos au-delà du cadre national}

Il convient de repréciser que seule la question de «l'organe-auteur», le dêmos ${ }^{62}$, de la constitution dans le contexte supranational qui se veut

54. On classe régulièrement la dénomination constitution dans la catégorie d'éléments non déterminants dans la définition formelle de la constitution. En ce sens, voir Marie-Anne Cohendet, Droit constitutionnel, Paris, Sirey, 2012, p. 70.

55. Francis Hamon et al., Droit constitutionnel, Paris, Sirey, 2012, p. 34.

56. Voir Théorie de la constitution, L. Deroche (trad.), Paris, PUF, 1993, p. 205.

57. Paolo Comanducci, «Épistémologie», art. cité, p. 23.

58. Voir Droit constitutionnel, Paris, Sirey, 2012, p. 71.

59. Voir «L'objet du droit constitutionnel... », art. cité, p. 63

60. Voir «Théorie générale des sources du droit constitutionnel», art. cité, p. 235.

61. Neil Walker, «Le constitutionnalisme multiniveaux», art. cité, p. 445 et suiv.

62. Cette question du dêmos est au cœur d'une nouvelle vision du droit: le droit démotique qui présente un double aspect national et international. Voir à ce propos 
plus ou moins métapolitique ${ }^{63}$ retiendra l'attention dans ce paragraphe. Il s'agira précisément de rechercher le peuple auquel on imputera la volonté générale dans ce cadre (1.1.1) et, en corollaire, le pouvoir constituant qui serait à l'origine de la «constitution internationale» (1.1.2).

\subsubsection{Un peuple au-delà du cadre étatique?}

L'absence d'un peuple européen est régulièrement présentée comme un handicap à l'établissement d'une constitution et même d'un pouvoir constituant communautaire ${ }^{64}$. L'idée se résume pertinemment dans une formule du juge Dieter Grimm : «Sans peuple, pas de Constitution ${ }^{65}$ ». La même critique est régulièrement formulée contre l'idée de constitution dans le cadre purement international. Celui-ci serait «dépourvu de la dimension symbolique-esthétique [...] [la] fonction essentielle de conserver la mémoire de l'être d'une communauté politique ${ }^{66} »$. De manière précise, on rappelle dans ce cadre l'absence "d'un corps central comme sujet/objet d'une constitution ${ }^{67}$ ». Une question excite la curiosité : quel est le critère retenu pour la qualification de la notion de peuple dans ce contexte? Le critère sociologique ou le critère juridique?

On sait qu'au sens sociologique le peuple est dénué de tout support juridique. C'est le souverain ${ }^{68}$. La doctrine dominante le situe hors de l'ordre constitutionnel ${ }^{69}$. Il serait, aux termes de l'article 21 paragraphe 3

Léopold Donfack Sokeng, Le droit des minorités et des peuples autochtones au Cameroun, thèse de droit, université de Nantes, 2001, p. 14 et suiv.

63. Neil Walker, «Le constitutionnalisme multiniveaux», art. cité, p. 449 et suiv.

64. "[I]l manque, pour faire de la "Constitution" européenne une vraie constitution un dêmos européen susceptible de s'auto-organiser par cet acte constituant, c'est-à-dire qui soit à la fois la source et le destinataire d'une constitution démocratique européenne», disait par exemple Olivier Jouanjan («Ce que donner une "Constitution à l'Europe" veut dire", Cités, vol. 1, n 13, 2001, p. 28).

65. Reproduite par Olivier Jouanjan, ibid., p. 28. Voir dans le même ordre d'idées, Miguel Herrerode Miñón, «Pour le peuple mais sans le peuple. Parallélismes entre la Constitution européenne et le constitutionnalisme philo-soviétique», dans La constitution et les valeurs, op. cit., p. 409 et suiv.

66. Anne Peters, «Reconstruction internationaliste du droit international...», op. cit., p. 364.

67. Gunther Teubner, «Constitutionnalisme sociétal et globalisation : alternatives à la théorie constitutionnelle centrée sur l'Etat», Revue juridique Thémis, vol. 29, $\mathrm{n}^{\circ}{ }_{3}$, 2005, p. 444.

68. Olivier Cayla, «L'obscure théorie du pouvoir constituant originaire » dans Denys de Béchillon et al. (coord.), L'architecture du droit, op. cit., p. 251.

69. Raymond Carré de Malberg, Contribution à la théorie générale de l'État, Paris, Dalloz, 2003, t. II, p. 485. 
de la déclaration universelle des droits de l'homme du 10 décembre 1948, le fondement de l'idée de constitution: d'où le rapport entre la dêmoskrátos (le pouvoir du peuple) et la constitution. Un rapport qui fait dire à Paolo Comanducci qu'il «ne peut y avoir de constitution sans démocratie ni de démocratie sans constitution ${ }^{70}$ ». En le situant au plan sociologique, si tel est le cas, le peuple dont on parle est-il perçu au sens spirituel ou matériel? À supposer qu'il soit pris au sens spirituel, doit-il nécessairement préexister à l'ordre juridique ainsi qu'il est réputé en Occident? Au sens matériel, un peuple européen de "race pure» a-t-il un intérêt scientifique dans un contexte où le cosmopolitisme ${ }^{71}$ est considéré comme l'élément fondamental de l'« ordre global ${ }^{72}$ »? À l'ordre international, la recherche de la dimension politique (empirique) conduirait à une analyse des sujets de droit international (États et organisations internationales) en tant que sujets constitutionnels ${ }^{73}$. Toutes ces préoccupations démontrent la place secondaire qu'occuperait la question dans le discours juridique. Une question qui pourrait finalement n'avoir de sens qu'à partir du principe de la légitimité construit par l'adhésion du peuple, « entité purement théorique » suivant le mot de Lucien Jaume ${ }^{74}$, à l'ordre juridique et à ses institutions ${ }^{75}$. Sans doute faudrait-il ici lire l'adhésion comme une caractéristique de l'effectivité (application par/contre les peuples, citoyens actifs ${ }^{76}$ ) du droit que les institutions supra-étatiques créent au-delà du cadre national, mais à son adresse. Dans ce cas, l'idée d'un constitutionnalisme suit celle d'une constitution établie comme expression d'un contrat social constaté plutôt que décidé ${ }^{77}$. L'hypothèse est celle d'un peuple (au sens sociologique et spirituel) formé à la faveur de l'attachement aux valeurs

70. Voir «Épistémologie», art. cité, p. 25.

71. L'idée que sous-tend le cosmopolitisme pourrait être appréhendée à partir de «la superposition des citoyennetés [...], la possibilité d'appartenance simultanée à plusieurs communautés politiques » (Mireille Delmas-Marty, Les forces imaginantes $d u$ droit, Paris, Seuil, t. III, 2007, p. 113).

72. Stéphane Pierré-Caps, «Le droit constitutionnel entre universalisme et particularisme ", dans Démocratie et liberté: tension, dialogue, confrontation. Mélanges offerts à Slobodan Milacic, Bruxelles, Bruylant, 2007, p. 218.

73. Gunther Teubner, "Constitutionnalisme sociétal et globalisation...», art. cité, p. 444.

74. Voir Michel Troper, «Le projet de Constitution européenne et la question du pouvoir constituant», Cités, vol. 1, n ${ }^{\circ}$ 13, 2001, p. 104.

75. Nicolas Belorgey, "Les origines de la légitimité non démocratique», Cités, vol. 1, $\mathrm{n}^{\mathrm{o}} 13,2001$, p. 67-79.

76. Michel Troper, «Droit constitutionnel et théorie générale de l'État», art. cité, p. 218.

77. Stéphane Pierré-Caps, «Le droit constitutionnel entre universalisme et particularisme», art. cité, p. 213 et suiv. 
communes (promotion de la démocratie, de l'État de droit, la garantie des droits de l'homme, etc. ${ }^{78}$ ). Jean-Louis Quermonne indiquait alors que :

[...] la construction européenne ne pourra disposer de fondations solides que si elle réussit, en s'appuyant sur le principe de subsidiarité, à établir un lien irréversible entre des peuples qui restent solidairement attachés à leurs histoires et à leurs singularités respectives, mais qui savent que dans un monde en extension et en crise permanente, ces valeurs ne pourront être sauvegardées que par la formation d'une union qui ne soit pas simplement une alliance, comme fut naguère le concert européen, mais une institution c'est-à-dire, soyons clair, un nouveau type de fédération ${ }^{79}$.

De fait, si on se place sur un plan purement juridique dans lequel le peuple n'existe pas en tant que donnée naturelle - thèse de Kelsen ${ }^{80}$ ou du moins pas avant la constitution comme l'a écrit Michel Troper ${ }^{81}$, le problème semble être résolu à partir de la pratique institutionnelle qu'on observe au-delà du cadre étatique. La constitution serait dès lors, ainsi que l'ont écrit Constance Grewe et Hélène Ruiz Fabri, une œuvre construite en amont du peuple dont la qualification y est liée ${ }^{82}$. On prendrait cette fois la légitimité, la souveraineté et le peuple en aval du phénomène juridique pour dire qu'il y a un ordre constitutionnel au-delà du cadre étatique, dès lors que les institutions produisent du droit à l'adresse des citoyens; un droit qui constitue pour eux un patrimoine juridique commun ${ }^{83}$. C'est la question de la gouvernance globale qui porte l’idée de légitimité au niveau supra-étatique.

À l'ordre international, la dynamique constitutionnelle du droit international tend à démontrer que la légitimité n'est pas liée à l'État, mais à la souveraineté du peuple capable de se déployer, avec ou sans l'intermédiation des organes étatiques classiques, devant les instances

78. Vlad Constantinesco, «Je t'aime, moi non plus! La société européenne en quête d'affection?", dans État, société et pouvoir à l'aube du XXI siècle. Mélanges pour François Borella, Presses universitaires de Nancy, 1999, p. 143 et suiv.

79. Voir Jean-Louis Quermonne, «L'adaptation de l'État à l'intégration européenne», RDP $1998, \mathrm{n}^{\text {os }}$ 5-6, p. 1420.

80. Théorie pure du droit, Charles Eisenman (trad.), Paris, Dalloz, 1962, p. 380-381.

81. Michel Troper, «Le projet de Constitution européenne et la question du pouvoir constituant», art. cité, p. 104.

82. Voir Droits constitutionnels européens, op. cit., p. 33 et suiv.

83. "[P]uisque nul ne peut aujourd'hui sérieusement contester que la Communauté européenne ne soit un ordre juridique propre et autonome, elle doit donc avoir une "constitution" ", concluait même Olivier Jouanjan ("Ce que donner une "Constitution à l'Europe" veut dire», art. cité, p. 29). 
internationales ${ }^{84}$. Au plan communautaire, dira Dominique Rousseau, «le vivre ensemble s'étend [...] aux peuples européens qui la constituent $^{85}$ ». Un autre auteur explique que le «sentiment d'appartenance [à la communauté] résulterait du "lien formel de la citoyenneté commune au même gouvernement politique ${ }^{86 ”}$ ". Ce faisant, la convention $\mathrm{EDH}$ serait par exemple un cadre fédérateur des ordres nationaux tout au moins ${ }^{87}$. Les influences de la CEDH sur la jurisprudence des États rentrent dans cette analyse ${ }^{88}$. La conclusion serait que la question d'une constitution européenne est réglée de manière inattendue ${ }^{89}$ - et que l'UE fonctionne déjà comme une sorte de fédéralisme ${ }^{90}$ coopératif suivant Jean-Louis Quermonne ${ }^{91}$ - pendant qu'on essaie de lui

84. Anne Peters, «Reconstruction constitutionnaliste du droit international...», art. cité, p. 372-373.

85. Dominique Rousseau, «Le nouvel horizon du droit constitutionnel», dans Renouveau du droit constitutionnel. Mélanges offerts à Louis Favoreu, Paris, Dalloz, 2007, p. 893 .

86. Katia Blairon, «La question démotique et la problématique d'une constitution européenne ", dans Réimaginer les frontières du droit constitutionnel, rapport présenté au Congrès international de droit constitutionnel, Athènes, 11-15 juin 2007, p. 2.

87. Charlotte Denizeau, Existe-t-il un bloc de constitutionnalité?, Paris, LGDJ, 1997, p. 117.

88. Il s'agirait d'une instance souveraine, du moins si on entend par instance souveraine une autorité en «mesure de faire valoir son droit au détriment de toutes les autres réalités sociales». Voir pour cette acception, Michaël Fœssel, «La souveraineté ou la part de l'irréductible», Esprit, $\mathrm{n}^{\circ} 281$, janvier 2002, p. 150.

89. On parle de la création d'une société européenne de droit public. Voir le numéro spécial $\left(\mathrm{n}^{0}{ }^{5}\right.$ ) de RFD comp. 2004, p. 671-672.

90. Marie-Claire Ponthoreau, Droits constitutionnel(s) comparé(s), Paris, Economica, 2010, p. 184 et suiv. "C'est [...] la théorie du fédéralisme qui a permis de souligner l'importance et l'intérêt de la prise en compte de la problématique internationaliste par les internistes», disait Michel Bélanger ("Considérations sur la "constitutionnalisation internationale"... ", art. cité, p. 44.

91. «L'adaptation de l'État à l'intégration européenne», art. cité, p. 1418 et suiv. On renverra également, de manière générale, à Jean Rideau qui développe la thèse d'un système fédéral à partir de la répartition des compétences (p. 481 et suiv.) et d'une double personnalité juridique interne et internationale au sein de l'union (p. 231 et suiv.). On notera aussi la capacité juridique reconnue à l'union en matière d'accords internationaux ou encore en matière (p. 239) dans Droit institutionnel de l'union et des communautés européennes, Paris, LGDJ, 2002. Jean-Denis Mouton conclurait "qu'il y a déjà en tout cas un phénomène politique suffisamment intéressant qui fait apparaitre des institutions politiques, qui gèrent des politiques communes, et qui connait l'amorce d'une citoyenneté de l'Union qui se superpose aux nationalités» («Crise et internationalisation de l'État: une place pour l'État multinational ? ", dans Olivier Audéoud et al. (dir.), L'État multinational et l'Europe, Presses universitaires de Nancy, 1997, p. 17). 
appliquer la symbolique du constitutionnalisme formel ${ }^{92}$ que décriait René Capitant ${ }^{93}$.

\subsubsection{Un pouvoir constituant supra-étatique?}

Le problème à ce niveau se situe sur l'autre pan de la question de la légitimité. L'hypothèse développée consiste à nier aux représentants des États un pouvoir constituant. On parle du « "déficit démocratique" de l'Union ${ }^{94} »$. La question est donc celle de la légitimité des organes communautaires en général et celle de ceux qui édictent les règles de forme constitutionnelle en particulier.

Cette question de la légitimité invite à un examen des mandats accordés aux «magistrats constitutionnels ${ }^{95}$ " de l'ordre extraétatique. Il est souvent rappelé que le mandat que détiennent les parlementaires européens n'en fait pas des constituants et même peut-être pas des législateurs. Le projet de constitution présenté par eux fut d'ailleurs considéré comme dénué «de mandat constitutionnel populaire ${ }^{96} »$. Cette limitation du mandat et de la souveraineté en général semble tout de même curieuse quand on sait que les membres du Parlement européen sont élus au suffrage universel ${ }^{97}$ au même titre que les parlementaires au

92. Nous renverrons aux développements d'Olivier Jouanjan, "Ce que donner une "Constitution à l'Europe" veut dire», art. cité, p. 29-35.

93. Voir "Le droit constitutionnel non écrit", dans Les sources des diverses branches $d u$ droit. Mélanges offerts à François Gény, Paris, Sirey, 1934, p. 3 et suiv.; « La coutume constitutionnelle», RDP $1979, n^{\circ} 4$, p. 962.

94. Jean-Louis Quermonne, «L'Union européenne : objet ou acteur de sa constitution? Essai sur la portée d'une politique institutionnelle à long terme», RF sc. pol. 2004, vol. $54, n^{\circ} 2$, p. 226.

95. Expression empruntée à Olivier Beaud d'après son article «Le Souverain », Pouvoirs, $\mathrm{n}^{\mathrm{o}} 67,1993$, p. 40.

96. Charlotte Denizeau, Existe-t-il un bloc de constitutionnalité?, op. cit., p. 124. La conclusion ne serait certainement pas la suivante à savoir que si «les autorités européennes peuvent produire des actes supérieurs aux lois parlementaires, ou bien la loi nationale n'est plus l'expression de la volonté générale ou bien le peuple n'est plus souverain » (Michel Troper, La théorie du droit, le droit, l'État, Paris, PUF, 2001, p. 320), mais bien plutôt que l'acte communautaire est, au même titre que «la loi nationale ", l'expression de la volonté générale produite par intermédiation - et non par le souverain qui n'existe pas dans l'ordre juridique.

97. Le principe est que la souveraineté nationale appartient au peuple qui l'exerce par l'intermédiaire de ses représentants ou par voie de référendum. Or, dans les faits, les eurodéputés au même titre que les députés nationaux (exemple pris de la France) sont élus au suffrage universel direct. Voir Jean-Louis Quermonne, «L'adaptation de l'État à l'intégration européenne», art. cité, p. 1406. 
sein des États qui jouissent des prérogatives de la souveraineté constituante ${ }^{98}$. Cela ne va pas sans quelques commentaires ${ }^{99}$. On soutiendra, par parallélisme, que dès lors que les eurodéputés tiennent leur légitimité du "peuple souverain", seule une contre-habilitation de même rang leur interdirait de statuer en tant que pouvoir constituant. Sur le plan international, la «légitimité constituante» est directement tenue de la constitution. Lorsqu'en effet le peuple, ou finalement l'État, proclame par l'intermédiation de la constitution son adhésion aux principes universels développés dans les déclarations et pactes internationaux, il s'engage dans un vaste système juridique dont les mécanismes sont définis par les instances internationales de promotion de ces valeurs.

Le second volet de la question de la légitimité se trouve dans l'importance accordée au juge constitutionnel dans l'ordre juridique national. La qualité de représentant du peuple constituant ${ }^{100}$ qui lui est reconnue pourrait bien se muer en un pouvoir constituant formel lui permettant d'introduire «sans bruit ${ }^{101}$ " des normes de référence dans une sorte de charte constitutionnelle que les citoyens pourront mobiliser, par contestation ou non, devant les instances (cours et commissions) supranationales. Cette remarque permet d'inverser les termes du débat.

$\mathrm{Si}$ en effet l'organisation et le fonctionnement des institutions audelà du cadre étatique promeuvent l'idée d'une constitution (au sens matériel tout au moins), il conviendrait d'admettre que celle-ci relève, formellement, d'une procédure coutumière. Des auteurs comme René Capitant n'avaient-ils pas tranché que la constitution est une règle essentiellement coutumière ${ }^{102}$ ? Olivier Jouanjan parle pour sa part

98. Cons. const., 2 sept. 1992, $\mathrm{n}^{\circ}$ 92-312 DC du (Traité sur l'Union européenne "Maastricht 2 ", cons. 19).

99. Stéphane Pierré-Caps tranche pour un «exercice commun $\mathrm{d}[\mathrm{u}]$ pouvoir constituant, qui peut être un pouvoir constituant européen exercé par les nations, parties constitutives de l'intégration politique européenne» ( $\left(\mathrm{La} \mathrm{V}^{\mathrm{e}}\right.$ République et la souveraineté », RDP $1998, \mathrm{n}^{\circ}$ 5/6, p. 1479.

100. Philippe Blachèr, Contrôle de constitutionnalité et volonté générale, op. cit., p. 185-195. On peut ainsi dire qu'il s'agit d'une autorité souveraine dès lors que «les normes issues de sa volonté sont incontestables et définitives» (Olivier Beaud, "Le Souverain», art. cité, p. 36.

101. Expression empruntée à Charlotte Denizeau, Existe-t-il un bloc de constitutionnalité?, op. cit., p. 125.

102. "La coutume constitutionnelle», art. cité, p. 962. Un autre auteur disait que «la structure de l'État est tributaire des lois et coutumes dites constitutionnelles; c'est elles qui impriment sa physionomie propre; il n'y a point de théorie de l'État à construire in abstracto » (Georges Renard, «Qu'est-ce que le droit constitutionnel. Le droit constitutionnel et la théorie de l'institution", dans Mélanges offerts à Raymond Carré de Malberg, op. cit., p. 485. 
d'une constitution présente «dans les traités des Communautés ${ }^{103}$ ». D'autres auteurs ont vu, dans le contexte européen toujours, un pouvoir constituant dérogatoire ou, pour être précis, un pouvoir constituant par évitement ${ }^{104}$, sans malheureusement approfondir la question ${ }^{105}$. La procédure coutumière signifie techniquement, dans ce cadre, la mise en accord des apparences avec la réalité juridique visée. Ce qui fondamentalement ne déroge pas à «l'opération constitutionnelle ${ }^{106}$ ». La question d'un pouvoir constituant au-delà du cadre étatique ne devrait donc pas être abandonnée à l'orthodoxie du constitutionnalisme des Lumières. Il n'y a de droit constitutionnel, au sens formel, que celui produit sous une forme documentaire précise : « Le droit positif n'est pas le droit posé par un législateur, il est le droit en vigueur, c'est-à-dire le droit appliqué, dont les prescriptions reçoivent généralement exécution dans une société donnée ${ }^{107}$.»

La question du pouvoir constituant est enfin liée à l'idée de souveraineté, disons au seul rapport à l'État ou à la constitution. Or, écrit Bertrand Badie, «[a]vec le processus de décolonisation, puis avec le processus de construction régionale et de mondialisation, de nouvelles constructions du concept de souveraineté ont vu le jour ${ }^{108}$ ». Ainsi la souveraineté désignerait la classe d' «arguments dont les autorités politiques ou juridictionnelles se servent effectivement dans toutes les sociétés modernes pour justifier la production de normes juridiques ${ }^{109}$ ». Elle désignerait un principe justificatif sur lequel repose le commerce juridique dans une société donnée. On pourrait postuler que la souveraineté nationale justifie les compétences des acteurs du système juridique et l'autorité des normes qu'ils produisent ou appliquent, autant

103. Voir «Ce que donner une "Constitution à l’Europe" veut dire", art. cité, p. 29.

104. Gaëlle Marti, Le pouvoir constituant européen, thèse de droit, université de Nancy II, 2008, p. 130-215 et 272-351.

105. On peut en effet regretter que l’auteur ait replongé dans les travers de la procédure classique des contextes dominés par le droit écrit notamment lorsqu'il trouve un pouvoir dérivé à la cour communautaire de justice du fait d'une supposée «subordination au pouvoir fondateur» (Ibid., p. 358 et suiv.) Peut-on en effet parler de pouvoir dérivé en l'absence d'un pouvoir originaire qui l'aurait formellement institué?

106. Expression empruntée à Olivier Jouanjan, «Ce que donner une "Constitution à l'Europe" veut dire», art. cité, p. 29 et suiv.

107. René Capitant, «Le droit constitutionnel non écrit », art. cité, p. 2 ; Georges Renard, "Qu'est-ce que le droit constitutionnel...", art. cité, p. 485.

108. Voir «La V $V^{\mathrm{e}}$ République et la souveraineté », art. cité, p. 1475.

109. Michel Troper, "Droit constitutionnel et théorie générale de l'État», art. cité, p. 220. 
que la vie internationale de l'État - puisqu'en réalité celui-ci «serait le titulaire d'une souveraineté intangible ${ }^{110}$ » moyennant la technique de la médiation constitutionnelle ${ }^{111}$-, et qu'elle pourrait aussi justifier un système juridique supra-étatique centralisé qui sous-tend l'idée d'une constitution. Cette idée de constitution correspond cette fois-ci au processus d'" engendrement d'un objet et [à] l'organisation de l'objet engendré ${ }^{112}$ ». La constitution se présente ainsi comme une fédération de systèmes d'énonciation du droit conçue par degré suivant la diversité et la pluralité des systèmes juridiques qu'elle rapproche ${ }^{113}$. Pour des auteurs comme Stéphane Pierré-Caps, l'intégration des particularismes dans la constitution nationale fait du droit constitutionnel « un phénomène universel ${ }^{114}$ ». Ce qu'il y a d'important est que les communautés et les individus peuvent désormais saisir les instances supranationales pour revendiquer le contenu de la souveraineté ${ }^{115}$. Celle-ci désigne « des compétences, des pouvoirs, des droits..., des attributs juridiques quels qu'ils soient, dont quelqu'un peut être titulaire ${ }^{116}$ ».

On retiendra donc, à la suite de Louis Favoreu, que l'idée d'une « constitution internationale » renvoie à «l'ensemble des normes constitutionnelles relatives au droit international (et au droit communautaire $^{117}$ ) ». La recherche d'un critère formel applicable à ce statut n'aura en définitive été qu'une hypothèse qui essaie de formuler les hésitations de la doctrine au moment où les constitutionnalistes sont interpellés sur la situation du monde ${ }^{118}$. Cette hypothèse est formulée à partir du

110. Marie-Anne Cohendet, Droit constitutionnel, op. cit., p. 149.

111. Emmanuelle Jouannet, «De la personnalité et la souveraineté de l'État dans la Constitution de 1958 », 27 p. (voir spécialement la p. 5) à consulter en ligne.

112. Voir «L'objet du droit constitutionnel : État, Constitution, démocratie», art. cité, p. 51.

113. C'est dans ce sens qu'Olivier Jouanjan présente la "Constitution européenne" en termes de «système constitutionnel complexe» («Ce que donner une "Constitution à l'Europe" veut dire», art. cité, p. 32).

114. Voir «Le droit constitutionnel entre universalisme et particularisme», dans Démocratie et liberté, op. cit., p. 217.

115. Bertrand Badie, « La V ${ }^{e}$ République et la souveraineté », art. cité, p. 1483.

116. Jean Combacau, «Pas une puissance, une liberté : la souveraineté internationale de l'État», Pouvoirs, nº 67, 1993, p. 49.

117. Voir « Le contrôle de constitutionnalité du traité de Maastricht et le développement du "droit constitutionnel international" ", art. cité, p. 41.

118. François Borella, "La situation actuelle du droit constitutionnel», RFD comp. 2012, $\mathrm{n}^{\circ} 89$, p. 4. 
constat que les instances supranationales décident "de la situation exceptionnelle ${ }^{119}$ »; précisément, « de la situation qu'aucune constitution n'est en mesure de prévoir ni d'anticiper ${ }^{120}$ ». N'est-ce pas à ce niveau qu'il faudrait rechercher le sens formel de la «constitution internationale» entendue comme la norme fondamentale de l'ordre juridique au-delà du cadre étatique?

\subsection{La «constitution internationale»: un ensemble de normes de référence}

Au sens formel, la constitution est aussi et surtout la norme fondamentale ${ }^{121}$ de l'ordre juridique. Cette caractéristique, somme toute la plus déterminante, se veut fédératrice et indépendante de la forme (écrite ou pas), de la procédure par laquelle elle a été produite et même de l'organe duquel elle tire ses origines. Il est évident que la procédure et l'organe originaires n'ont pas de support juridique. La forme elle-même importe peu puisqu'en Grande-Bretagne - contexte où les trois principes sont faiblement exigés ${ }^{122}$ - comme aux États-Unis, pays marqué par l'empreinte écrite, la liberté politique est le fondement du régime constitutionnel ${ }^{123}$. L'idée de norme, dans ce cas, est ancrée, non sur les critères formels précédemment énumérés, mais sur le lien causal entre la liberté politique et l'organisation de la société ${ }^{124}$.

Si elle est donc la norme fondamentale ou fondatrice, la constitution mérite d'être vue comme un tout déduit des différents énoncés - indépendamment de leur origine - établissant les règles et procédures auxquelles est soumis l'ensemble des acteurs du système juridique. L'intérêt poursuivi est la préservation de la philosophie fondatrice de l'ordre global (la liberté politique par exemple): la norme fondamentale. De plus, les règles qui en dérivent et qu'on peut isoler parce que propres à la mise en place d'un ordre autonome peuvent être mobilisées (devant

119. Expression empruntée à Carl Schmitt, Théologie politique, J. L. Schlegel (trad.), Paris, Gallimard, 1988, p. 15.

120. Michaël Fœssel, «La souveraineté ou la part de l'irréductible», art. cité, p. 155.

121. L'idée se résume par la primauté hiérarchique et l'impact sur les autres normes. La constitution constitue ainsi le domaine de validité des normes juridiques (MarieAnne Cohendet, Droit constitutionnel, op. cit., p. 70-71).

122. Vlad Constantinesco et al., Droit constitutionnel, op. cit., p. 199.

123. On renverra à l'œuvre d'Émile Boutmy, Éléments d'une psychologie politique du peuple américain. La nation. La patrie. L'État. La religion, Paris, A. Colin, 1902, p. 95 et suiv. et Essai d'une psychologie politique du peuple anglais au XIX ${ }^{e}$ siècle, Paris, A. Colin, 1916, p. 298 et suiv.

124. Michel Troper, Le droit et la nécessité, Paris, PUF, 2011, p. 13. 
les instances nationales et supranationales à l'instar de la Cour constitutionnelle internationale qui retient désormais l'attention des spécialistes) par les membres (citoyens) d'une société politique sans frontière. La " constitution internationale" réalise sous cet angle une unité de règles (1.2.1); mais bien plus, un conglomérat de cultures, d'histoires et donc de systèmes juridiques ${ }^{125}$ : une norme fondamentale en somme (1.2.2).

\subsubsection{Un corps de règles communes}

La « constitution internationale » serait, ainsi que l’a écrit Alfred Verdross, le point de départ de toutes les règles juridiques supra-étatiques ${ }^{126}$. L'idée de «constitution internationale » s'insère ainsi dans une conception du droit que Louis Favoreu qualifiait de "Constitution du droit». Elle est formée par l'ensemble des «normes constitutionnelles qui se situent à la charnière entre l'ordre international et l'ordre interne ${ }^{127}$ " et mobilise les autorités des différents ordres juridiques en jeu. La "constitution internationale» procéderait ainsi d'un ensemble de «règles d'autoproduction ${ }^{128}$ ». On pourrait les présenter en deux catégories: une première nationale et une seconde supranationale ${ }^{129}$.

Rentrent dans la première catégorie, les règles de procédure qui visent à introduire la règle supranationale dans l'ordre interne et les différents mécanismes de la codification du droit international ${ }^{130}$ qui placent à leur

125. La corrélation entre l'histoire et l'ordre juridique a été parfaitement établie par Georg Jellinek dans L'État moderne et son droit, G. Fardis (trad.), Paris, A. Fontemoing, 1904, t. I, p. 66 et suiv.

126. Cité par Pascal Charlemagne Nyamnding Messanga, « Peut-on envisager la translation du concept de la constitution dans le cadre juridique international ? ", art. cité, p. 88.

127. Voir « Le contrôle de constitutionnalité du traité de Maastricht et le développement $\mathrm{du}$ "droit constitutionnel international" », art. cité, p. 41 et 58. L'auteur parle de "normes de droit positif tirées du droit international ou du droit communautaire et européen et que l'on peut dénommer - faute de mieux - des normes internationales ou supranationales supraconstitutionnelles». Voir «Souveraineté et supraconstitutionnalité », Pouvoirs, $n^{\circ} 67,1993$, p. 74.

128. Gunther Teubner, "Constitutionnalisme sociétal...», art. cité, p. 455.

129. Jean Combacau, «Sources internationales et européennes du droit constitutionnel », art. cité, t. I, p. 419-439.

130. Il est écrit à ce propos que «les traités se caractérisent par le fait qu'ils mettent en cause deux ordres juridiques différents: les règles relatives à leur élaboration et à leur exécution relèvent autant du droit international que du droit constitutionnel propre à chacun des États engagés dans le commerce international et "il est bien difficile d'imaginer une hypothèse dans laquelle la portée du traité dans un ordre juridique serait sans répercussions sur le sort de l'autre” ». Voir Francis Rigaldies 
tour l'autorité publique dans une perspective de dédoublement fonctionnel $^{131}$ ou d'administration de réseau ${ }^{132}$. Certains auteurs parleraient de «droit national d'intégration ».

C'est encore Louis Favoreu qui se demandait si « en signant les traités institutifs les gouvernements ${ }^{133}$ " s'engageaient à tout ce qui en allait découler. Il s'interroge sur l'avenir de l'État et de la souveraineté nationale notamment. Il ne met pas moins en relief le caractère supranational de la ratification et donc de la formation du droit au-delà du cadre étatique. Pour sa part, Michel Bélanger pensait que l'adoption des normes justifiant la pratique du droit international constitue «le pilier principal de la constitutionnalisation internationale ${ }^{134}$ ». Il suivrait alors que les dispositions constitutionnelles, à l'exemple de l'article 117 de la Constitution du Mali par lequel cet État «peut conclure avec tout État africain des accords [...] comprenant abandon partiel ou total de souveraineté en vue de réaliser l'unité africaine ", inscrivent l'État dans la perspective de la constitutionnalisation du droit international dès lors que les instances qui suivent l'ordre juridique (consenti par voie constitutionnelle) obligent l'ordre juridique national dans son ensemble. Les règles constitutionnelles qui engagent internationalement l'État seraient donc des règles de référence pouvant être interpellées pour rappeler une obligation supranationale ${ }^{135}$.

La seconde catégorie est formée de règles supranationales. Les déclarations de 1789 et de 1948 , les pactes internationaux de 1966, la charte africaine des droits de l'homme et des peuples du 27 juin 1981 obligent les juridictions nationales à trouver du droit au sein des instruments juridiques à caractère international voire universel ${ }^{136}$. On a vu plus haut que, les textes communautaires ou universels relatifs aux libertés et droits

et al., «Le juge interne canadien et le droit international », Les Cahiers du droit, vol. 21, $\mathrm{n}^{\circ}$ 2, 1980, p. 313-314.

131. Georges Scelle, «Le droit constitutionnel international», op. cit., p. 513-515.

132. Jean-Louis Quermonne, "L'adaptation de l'État à l'intégration européenne», art. cité, p. 1416 et suiv.

133. Louis Favoreu, «Le contrôle de constitutionnalité du traité de Maastricht et le développement du "droit constitutionnel international" ", art. cité, p. 61.

134. Voir «Considérations sur la "constitutionnalisation internationale"...», art. cité, p. 46.

135. Hermann Mosler, «L'application du droit international public par les tribunaux nationaux ", RCADI 1957, $n^{\circ}$ 91, p. 619 et suiv.

136. Brusil M. Metou, «Le moyen de droit international devant les juridictions internes en Afrique : quelques exemples d'Afrique noire francophone ", Revue québécoise de droit international, vol. 1, $\mathrm{n}^{\mathrm{o}} 22,2009$, p. 129 et suiv. 
fondamentaux constituent de véritables chartes constitutionnelles ${ }^{137}$. Keba Mbaye disait de la déclaration de 1948 que, bien que n'étant pas « un instrument parfait [elle] exprime le consensus universel sur un minimum de principes et de règles relatifs aux droits de l'homme, et constitue aujourd'hui l'expression de la conscience de l'ensemble des États relativement à la dignité humaine ${ }^{138} »$. Pour Maurice Glèlè Ahanhanzo, la charte africaine serait « une loi fondamentale, une constitution [...] porteuse d'un projet de société fondé sur le règne et la prééminence du droit $^{139}$ ». D'autres auteurs y voient une règle fédératrice des sociétés occidentales et africaines ${ }^{140}$. Elle permettrait à l'Afrique de concilier ses valeurs endogènes avec l'impératif universel de la promotion des droits de l'homme. C'est ce qui fait dire à Keba Mbaye que les États africains, au même titre que les autres, "ont une obligation erga omnes vis-à-vis de la communauté internationale en matière de droits de l'homme ${ }^{141}$ ».

Il en ressort que la problématique des droits fondamentaux mobilise la communauté internationale dans son ensemble; que, dès lors, elle ne pourrait faire l'objet d'un traitement régional ou sous régional allant dans le sens d'un contra legem. N'est-ce pas là l'un des multiples sens qu'on pourrait donner à la notion de rigidité constitutionnelle ${ }^{142}$ ? Quoi qu'il en soit, il est établi que la concordance des jurisprudences dans la promotion des droits fondamentaux conduit à l'élaboration d'un corpus constitutionnel ${ }^{143}$, «par le jeu d'emprunts unilatéraux ou réciproques, par suite de convergences fortuites ou délibérées, les différents systèmes, tant nationaux que supranationaux, de protection des droits de l'homme façonnent au travers de leurs jurisprudences d'application respective un ensemble de principes et de règles qui matériellement s'apparente à une charte constitutionnelle», explique Jean-François Flauss ${ }^{144}$. On peut

137. Le doyen Favoreu parlerait $\mathrm{d}^{\prime}$ ' $u$ un fonds commun de principes transnationaux qui peuvent - s'il en est besoin - compléter ou conforter [l']interprétation de la Constitution» ( «Le contrôle de constitutionnalité du traité de Maastricht et le développement du "droit constitutionnel international" ", art. cité, p. 75).

138. Voir Les droits de l'homme en Afrique, Paris, Pedone, 2002, p. 98.

139. Voir «Introduction à la Charte des Droits de l'Homme et des Peuples», dans Organisation de l'Unité africaine, études offertes à Claude-Albert Colliard, Paris, Pedone, 1984, p. 517.

140. Alioune Badara Fall, «La Charte africaine des droits de l'homme et des peuples: entre universalisme et régionalisme ", Pouvoirs, $\mathrm{n}^{\circ}$ 129, 2009, p. 77 et suiv.

141. Voir Les droits de l'homme en Afrique, op. cit., p. 187.

142. Michel Bélanger, "Considérations sur la "constitutionnalisation internationale"...», art. cité, p. 48-49.

143. Jacques Pertek, Droit des institutions de l'union européenne, op. cit., p. 464 et suiv.

144. Reproduit par Charlotte Denizeau, Existe-t-il un bloc de constitutionnalité?, op. cit., p. 122. 
ainsi retenir au moins deux principes à caractère pédagogique: d'une part, les droits fondamentaux exigent la fondamentalisation du droit ${ }^{145}$ qui, d'autre part, requiert l'idée d'une "constitution internationale " entendue comme un assemblage de systèmes juridiques nationaux et supranationaux.

\subsubsection{Une fédération de systèmes juridiques}

La norme fondamentale opère une synthèse entre les différents systèmes juridiques antagonistes qui composent une société politique. Cela est vrai dans un ordre centralisé, l'État traversé par un pluralisme social et donc juridique. On a en France l'exemple de la spécificité kanake et néocalédonienne en général conciliée à l'ordre juridique par la technique de la constitution ${ }^{146}$. La constitution, au sens formel, serait donc au surplus un instrument de cohésion ${ }^{147}$. Au-delà de l'État, l'idée renverrait à la mise en forme d'une unité juridique à partir des différents ordres nationaux qui présentent autant de différence que leur nombre. Alioune Badara Fall prenant l'exemple du principe universel (norme fondamentale) de la promotion des droits fondamentaux fait remarquer le contraste entre l'universalité des droits de l'homme et le pluralisme juridique en Afrique $^{148}$. La question qui vient à l'esprit est celle $d u$ comment concilier les différentes approches des droits de l'homme, sauf à les présenter comme un tout qui constituerait un grand marché de dupes $^{149}$. Si on admet en effet que les droits de l'homme constituent

145. François Luchaire, "Le Traité d'Amsterdam et la Constitution », RDP 1998, $\mathrm{n}^{\circ}$ 2, p. 340 et suiv.

146. Titre XVII de la Constitution de 1958 ou décision n ${ }^{\circ}$ 99-410 DC du 15 mars 1999 (Loi organique de la Nouvelle-Calédonie).

147. Dominique Rousseau, "Le droit constitutionnel, droit d'harmonie», Actes des journées de la Société de législation comparée, 1995, p. 337.

148. Voir «Universalité des droits de l'homme et pluralité juridique en Afrique. Analyse d'un paradoxe", dans La constitution et les valeurs, op. cit., p. 359 et suiv.

149. Pareille conclusion a déjà été posée à propos de l'intégration des États européens postcommunistes dans l'Europe démocratique : «l'intégration des États d'Europe centrale et orientale dans la galaxie des organisations européennes relève "bien plus d'une logique d'imposition que d'une logique d'adaptation. L'alignement de ces États sur les standards normatifs européens et la démocratisation de leurs institutions politiques" ont pris "l'allure d'une marche forcée, dans laquelle certains ont pu discerner une forme d'impérialisme démocratique de l'Europe de l'Ouest, un véritable droit d'ingérence au regard des principes de souveraineté et d'autonomie constitutionnelle des États" " (Stéphane Pierré-Caps, "Le droit constitutionnel entre universalisme et particularisme», art. cité, p. 216. Italiques de l'auteur). 
le domaine privilégié de la construction d'une «conscience juridique internationale ${ }^{150}$ », il faut néanmoins se demander comment on parvient à formuler cette synergie de droits ${ }^{151}$.

La question est celle de la valeur universelle ou internationalisée de la déclaration de 1948 par exemple ${ }^{152}$. Prenons le principe d'égalité qu'elle proclame et qui présente une société indifférenciée construite autour de la citoyenneté, une identité univoque. Ce principe serait incommode au sein des sociétés plurales traversées par l'hétérogénéité ${ }^{153}$. Keba Mbaye dira que la "perception volontairement universaliste et éthérée de la citoyenneté ${ }^{154}$ » masque le désir d'égalité qu'éprouvent les Africains depuis la nuit coloniale ${ }^{155}$. À chaque milieu social correspondant un système juridique (on parle, pour rester dans le domaine qui nous intéresse dans l'immédiat, de droits de l'homme africains ${ }^{156}$, amérindiens ${ }^{157}$, arabo-islamiques ${ }^{158}$ ou encore européens ${ }^{159}$ ), il conviendrait de comprendre le mouvement de la constitutionnalisation internationale comme concourant à la réalisation de la synthèse recherchée face à une telle disparité ${ }^{160}$. Les techniques constituantes qui, à l'ordre interne au moins, opèrent la symétrie à partir des courants juridiques opposés participent d'une vision intégrée du droit ${ }^{161}$.

150. Expression empruntée à Christopher Jamin, «La construction de la pensée juridique française », art. cité, p. 504.

151. Nous renverrons aux développements de Léopold Donfack Sokeng dans "Mondialisation et droits de l'homme en Afrique», Revue camerounaise d'études internationales, $\mathrm{n}^{\mathrm{o}} 1,2007$, p. 103-132.

152. Gérard Cohen-Jonathan, «Les droits de l'homme, une valeur internationalisée ", Droits fondamentaux, $\mathrm{n}^{\mathrm{o}}$ 1, 2001, p. 157-159.

153. Il est en effet écrit: "Dans le monde des sociétés occidentales contemporaines, hautement complexes et différenciées, ou la cité politique est toujours concurrencée dans la vie des hommes par d'autres regroupements tels que la religion, la secte, communauté ethnique, la famille, la profession ou la “bande", le citoyen n'est sûrement pas le premier pôle d'identification des individus» (Olivier Duhamel et al., Dictionnaire constitutionnel, Paris, PUF, 1992, p. 145).

154. Alian-Didier Olinga, «La protection des minorités en droit public camerounais», Revue africaine de droit international et comparé, 1998, p. 283.

155. Voir Les droits de l'homme en Afrique, op. cit., p. 202.

156. Voir la conférence afro-asiatique de Bandoeng du 24 avril 1955.

157. Voir la convention américaine relative aux droits de l'homme du 22 novembre 1969.

158. Voir la déclaration islamique universelle des droits de l'homme de 1981.

159. Voir la charte des droits fondamentaux de l'Union européenne.

160. Maurice Glélé-Ahanhanzo, Introduction à l'organisation de l'unité africaine et aux organisations régionales africaines, Paris, LGDJ, 1986, p. 80.

161. Michel Bélanger, "Considérations sur la "constitutionnalisation internationale"...», art. cité, p. 43 . 
Par «constitutionnalisation internationale", il faut voir le mouvement d'institutionnalisation des «mécanismes d'autorestriction contre l'expansion d'un secteur social hégémonique ${ }^{162}$ ». L'idée de monisme qui suivrait ce processus désigne la mise en application centralisée des normes à vocation universelle sécrétées par les différentes institutions nées de cette opération. C'est dans cette perspective qu'Alain Pellet dirait du droit des Nations unies qu'il est «constitutionnalisé ${ }^{163} »$. On sait que la performance des instances supranationales obligerait, soutient Jean Combacau, «l'État à introduire, maintenir ou abroger telle norme constitutionnelle particulière dans son ordre juridique ou lui interdirait de la faire ${ }^{164} »$. Gunther Teubner en conclurait que l'idée de "constitution internationale» est le fruit d'une construction progressive, d'une agrégation «des sous-systèmes autonomes de la sociétémonde ${ }^{165}$ ». L'idée d'une «constitution internationale» recherchée se trouve assurément dans la réponse «à la question de l'opposabilité des êtres légaux résultant du jeu des modes de production qu'[elle] comporte $^{166}$ ». La «constitution internationale», au sens formel du terme, serait ainsi un ensemble de normes tirées de différentes cultures juridiques, de différents ordres juridiques. Il convient sans doute de relever l'idée d'État, «dont les diverses institutions juridiques nationales sont les avant-postes », développée par Jean-François Kervegan ${ }^{167}$. Cette idée rejoint la thèse du phénomène intersocial antérieurement développé par Georges Scelle et caractérisé par la "supériorité nécessaire de la solidarité globale sur les solidarités locales ${ }^{168} »$. Le propos traduit une certaine hiérarchie au sein des différents ordres qui composent cette synergie formulée à partir du vœu d'une « constitution internationale». Une telle exigence ne peut être recherchée qu'à partir de l'aspect matériel de la constitution.

162. Gunther Teubner, «Constitutionnalisme sociétal...», art. cité, p. 449.

163. Voir «Constitutionnalisation du droit des Nations unies ou triomphe du dualisme?», RMCUE 2009, $n^{\circ}$ 529, p. 416.

164. Voir «Sources internationales et européennes du droit constitutionnel», art. cité, p. 421.

165. Voir "Constitutionnalisme sociétal...», art. cité, p. 446.

166. Jean Combacau et al., Droit international public, Paris, Montchrestien, 2010, p. 18.

167. Voir "Souveraineté, État de droit, supra-nationalité : un rapport contradictoire?", art. cité, p. 565.

168. Georges Scelle, «Le droit constitutionnel international», art. cité, p. 511. 


\section{L'aspect substantiel de la « constitution internationale»}

La constitution désigne, au sens matériel, l'ensemble des règles qui se rapportent à l'organisation des pouvoirs et à la garantie des droits et libertés ${ }^{169}$. La formule reprend l'énoncé de l'article 16 de la déclaration de 1789: "Toute société dans laquelle la garantie des droits n'est pas assurée ni la séparation des pouvoirs déterminée, n’a point de Constitution ». Elle pose l'exigence - sans rapport à l'État - d'une constitution "digne de ce nom ${ }^{170}$ ", en détermine les principes directeurs. S'agit-il pour autant d'une définition? Le moins que l'on puisse dire est que l'énoncé «se réfère à», mais sans «signifier». En d'autres termes, il ne présente pas les propriétés qui structurent l'objet censé être encadré ${ }^{171}$. Bien plus, la formule renvoie à l'idéologie révolutionnaire et, de ce point de vue, serait inappropriée à l'analyse scientifique parce qu'empreinte de subjectivité ${ }^{172}$. On pourrait certes la considérer comme le principe - philosophique, politique ou moral - fondateur de l'idée de constitution au sens moderne du terme ${ }^{173}$, mais la notion de constitution est ailleurs. Pour une part, elle est inséparable de l'ordre juridique. Pour une autre, elle renvoie au système de production de normes ${ }^{174}$. Et c'est en considération de ces impératifs de la science du droit qu'on définit la constitution, au sens matériel, comme «l'ensemble des normes de production de normes générales et abstraites ${ }^{175}$ ».

169. Vlad Constantinesco et al., Droit constitutionnel, op. cit., p. 199.

170. Expression empruntée à Francis Hamon et al., Droit constitutionnel, op. cit., p. 352.

171. Pierre Brunet, «Alf Ross et la conception référentielle de la signification en droit ", Droit et société, 2002, $\mathrm{n}^{\circ}$ 50, p. 26.

172. On ne saurait à cet effet isoler le droit constitutionnel révolutionnaire « de son contexte politique et social». Voir à ce propos Olivier Jouanjan, « Lorenz von Stein et les contradictions du mouvement constitutionnel révolutionnaire», AHRF, $\mathrm{n}^{\mathrm{o}} 328,2002$, p. 173. Il est certes défendu l'idée que le droit révolutionnaire "dépasse évidemment la seule organisation "française" du pouvoir et se présente comme un modèle de l'organisation de l'État (Éric Maulin, "Carré de Malberg et le droit constitutionnel de la Révolution française », $A H R F, \mathrm{n}^{\circ} 328,2002$, p. 10.), mais la théorie générale de l'État qui suit cette conception n'est-elle pas néanmoins une théorie bâtie sur une seule expérience historique? Voir à ce propos Michel Troper, Pour une théorie juridique de l'État, op. cit., p. 8-9.

173. Ce sens est pris en opposition à l'idée développée par Aristote à partir de la constitution d'Athènes formée des 158 textes organisant le pouvoir dans les cités grecques.

174. Hans Kelsen, «La garantie juridictionnelle de la Constitution (La justice constitutionnelle)», RDP 1928, $n^{\circ}$ XLV, p. 205.

175. Louis Favoreu et al., Droit constitutionnel, Paris, Dalloz, 2009, p. 74. 
Si en effet la constitution renvoie, au sens matériel, à l'organisation d'une société libérale (dixit art. 16 DDHC), il ne s'agit pas d'une donnée naturelle qu'on pourrait enfermer au sein de la seule cité-État ${ }^{176}$. Des auteurs comme Dominique Rousseau font observer que la construction du droit au-delà du cadre national oblige les constitutionnalistes à

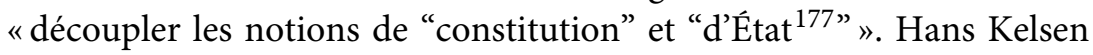
avait pour sa part rapproché les deux concepts sous la formule d'ordre juridique $^{178}$, une unité hiérarchisée de normes ${ }^{179}$. Chez Santi Romano, cet ordre correspond à la «manifestation d'unité de la société dans laquelle le droit s'affirme» en tant qu'institution ${ }^{180}$. Les deux courants (normatif et institutionnel) apparemment opposés ${ }^{181}$ illustreraient l'idée d'une «constitution internationale » dès lors qu'elle désigne matériellement un ensemble de règles poursuivant, non pas plusieurs, mais une norme fondamentale à un ordre juridique auquel on imputerait l'action des acteurs qui interviennent dans ce cadre. L'aspect matériel de la «constitution internationale » entretient l'idée d'un ordre juridique autonome ${ }^{182}$ marqué par l'indépendance de ses règles et par leur capacité à régir les principes essentiels d'une société sans frontières. On pourrait emprunter à Dominique Turpin l'idée selon laquelle l'aspect matériel de la «constitution internationale» désigne «l'internationalisation des exigences de l'État de droit et de la production des normes impératives ${ }^{183}$ ". Elle poursuit la fondamentalisation du droit au-delà du cadre national (2.1), un système autonome, avec ses acteurs et ses sujets, une société régie par une kyrielle d'institutions unifiées par la technique juridique (2.2).

176. Voir dans le même sens Pascal Charlemagne Nyamnding Messanga, «Peut-on envisager la translation du concept de la constitution dans le cadre juridique international ?», art. cité, p. 77-81.

177. Voir Dominique Rousseau, «Le nouvel horizon du droit constitutionnel », art. cité, p. 893 .

178. Voir Théorie pure du droit, op. cit., p. 378 et suiv.

179. Voir Théorie du droit international public, RCADI 1953, $\mathrm{n}^{\circ} 84$ : «Un ordre juridique n'est pas un groupe plus ou moins fortuit de normes. Il doit être conçu par la science du droit comme un système de normes placées dans un rapport réciproque, où les unes sont subordonnées aux autres» (p. 6).

180. Voir Santi Romano, L'ordre juridique, L. François et P. Gothot (trad.), rééd. Paris, Dalloz, 2002, p. vii.

181. Phocion Francescakis disait de Santi Romano qu'il est «l'anti-Kelsen» (Ibid., p. vi).

182. Michel Troper, «La constitution comme système juridique autonome», Droits, $\mathrm{n}^{\mathrm{o}} 35,2002$, p. $63-78$.

183. Voir «Mondialisation et normes juridiques. Pour un nouveau contrat social global », dans Démocratie et liberté, op. cit., p. 445. 


\subsection{Une «fondamentalisation» du droit au-delà du cadre national}

La «fondamentalisation» est un néologisme qui intègre le discours juridique pour rendre compte non pas de la qualité d'une règle ou d'une espèce de droits ${ }^{184}$, mais de la détermination juridique d'un ensemble de prérogatives qu'on qualifie de droits fondamentaux. Au-delà de l'ordre national, ce mouvement correspondrait aux prestations juridiques qui concourent à l'énonciation du régime des droits et libertés fondamentaux, pour rester dans la terminologie d'usage ${ }^{185}$. La fondamentalisation désigne le système de production et de promotion des règles essentielles de la société. Ce système élève la question de l'énonciation du droit au-delà du cadre étatique en opposant les différents ordres juridiques interpellés dans ce contexte (2.1.1). Il serait dès lors intéressant d'étudier comment le système positif de la production des normes - au sens kelsénien du terme ${ }^{186}$ - pourrait obliger (juridiquement) les acteurs à adopter un comportement conforme au protocole de promotion du ou des principe(s) directeur(s) de l'ordre global que leurs actions règlent (2.1.2).

\subsubsection{Norme et système d'énonciation du droit au-delà du cadre national}

Le concept de norme prend en compte deux aspects en théorie du droit. On a d'abord l'hypothèse d'une norme fondamentale ou fondatrice. C'est le signifiant ou l'idéologie - la promotion des droits de l'homme et de la séparation des pouvoirs par exemple - qui détermine la structure de l'ordre juridique pris globalement. Cette norme acquiert, rétroactivement, une valeur juridique puisqu'elle se lit dans la pratique du droit. Hans Kelsen l'un des promoteurs de cette idée écrit que «la norme fondamentale opère institution de l'élément fondamental des opérations de création du droit ${ }^{187}{ }$. C'est dire qu'au départ la norme fondamentale est juridiquement indéterminée. Il s'agit dans le meilleur

184. Qu'il nous soit permis de renvoyer à une précédente étude: Rodrigue Ngando Sandjè, "Le contentieux environnemental au Cameroun : entre tradition et modernité », dans Pauline Milon et al.(dir.), Révolution juridique, révolution scientifique, vers une fondamentalisation du droit de l'environnement?, Aix-en-Provence, Presses universitaires d'Aix-Marseille, 2014, p. 48-52.

185. Muriel Fabre-Magnan, Introduction au droit, Paris, PUF, 2014, p. 71.

186. La norme désigne, disait Hans Kelsen, «un commandement, un ordre, une prescription » (Théorie générale des normes, Paris, PUF, 1996, p. 1).

187. Voir Théorie pure du droit, op. cit., p. 263. 
des cas d'un énoncé qui ne rassemble pas moins d'un faisceau d'indices souvent épars, voire contradictoires. Des auteurs comme Gérard Timsit lui préfèrent un «système normatif à logique duale (au moins duale) dans lequel, au lieu que tout découle d'une norme suprême unique, se concurrencent, et parfois se contrarient, les principes fondateurs qui commandent à sa construction et son fonctionnement ${ }^{188} »$. Mais comment entreprendre, au sein d'un ordre centralisé, la concurrence entre les principes fondateurs du système juridique? Faut-il y voir un illogisme de la part du constituant? Prenons l'exemple du principe de l'indivisibilité au cœur de l'idée de république en France ${ }^{189}$. Il rassemble une pluralité d'indices qui se regarde différemment ${ }^{190}$ selon qu'on se réfère à une décision du Conseil constitutionnel (décision $\mathrm{n}^{0}$ 91-290 DC du 9 mai 1991) relative au statut de la Corse ou à la détermination constitutionnelle (art. 72-3 de la Constitution de 1958, décision 99-410 DC du 15 mai 1999) de la spécificité néo-calédonienne dans l'agencement de cette République indivisible. Contraste? N'est-ce pas plutôt la preuve que la norme fondamentale est juridiquement voire sémantiquement indéterminée? Si tel est le cas, on le suppose du moins, on conviendra que son sens (du point de vue juridique du moins) se découvre au gré des différentes interprétations qu'en font les acteurs du système juridique. Dès lors, la ou les norme(s) qu'ils produisent donne un second sens au mot: le(s) signifié(s) des principes fondateurs du système.

En ce sens, dira précisément Éric Millard, la norme est la «"signification d'un acte de volonté": un acte par lequel quelqu'un veut que quelque chose "doit avoir lieu ${ }^{191}$ " ». Elle exprime un commandement et concourt à la détermination, selon chaque espèce, du sens de la norme fondamentale. De là naît l'une des questions les plus débattues en théorie de droit: si une norme désigne "un commandement, un ordre, une prescription $^{192}$ ", comment peut-on dès lors hiérarchiser les normes du même système? Comment précisément mesurer les commandements? $\mathrm{Y}$ aurait-il une hiérarchie entre deux commandements $\mathrm{A}$ et A' déduits de la même norme fondamentale? Y aurait-il au sein du même ordre juridique un commandement $A$ et un semi-commandement A'? L'idée

188. Voir «L'ordre juridique comme métaphore», Droits, $\mathrm{n}^{\circ} 33$, 2001, p. 6-7.

189. François Luchaire, Le Conseil constitutionnel, t. III : L'État, Paris, Economica, 1999, p. 6.

190. Stéphane Pierré-Caps, «Le Conseil constitutionnel et la question du demos », dans Renouveau du droit constitutionnel, op. cit., p. 392.

191. Voir «Qu'est-ce qu'une norme juridique?», Cah. Cons. const. 2006, $n^{\circ}$ 21, p. 89.

192. Hans Kelsen, Théorie générale des normes, op. cit., p. 1. 
d'une hiérarchie des normes est sans doute une représentation déformée de la réalité ${ }^{193}$.

Kolb Robert en développe quelques aspects à partir des différentes articulations de la norme impérative de droit international (jus cogens) dont on dit qu'elle promeut une «constitutionnalisation normative internationale ${ }^{194} »$. L'auteur met en relief un potentiel conflit entre le nonrecours à la force et l'impératif de la protection des droits fondamentaux en faisant remarquer qu'une "norme n'est pas nécessairement impérative en bloc ${ }^{195}$ ». Il présente l'ordre juridique comme un objet sécable dont les particules seraient opposables. L'idée serait la même que celle précédemment développée selon que l'impératif ${ }^{196}$ - «expression linguistique d'un "commandement" » d'après Kelsen ${ }^{197}$ - serait un sphinx qui, disait Gabriel de Vareilles-Sommières dans un contexte différent, conduit à un illogisme ${ }^{198}$. Or il apparait que l'hétérogénéité du contenu de la norme impérative en droit international comme la diversité d'objets qui composent la norme fondamentale en général doivent leur coordination à l'action des acteurs du système juridique. Ces acteurs se rencontrent dans la promotion d'une valeur, les droits fondamentaux par exemple qui ont posé la base de "l'impératif juridique» en droit international $^{199}$. Cette hypothèse aurait suffi à rassurer la CJUE par rapport à une éventuelle adhésion de l'Union à la CEDH. Les expressions redoutées comme «standards de protection des droits fondamentaux plus élevés ${ }^{200}$ " (avis $2 / 13$, point 184 ) constitueraient ainsi une simple rhétorique sans véritable implication juridique à partir du moment où la gouvernance des droits fondamentaux permet d'établir une chaîne de commandement dans la production des normes à partir du dialogue entre les juges des trois différents ordres (États membres, UE et parties

193. Gérard Timsit, «L’ordre juridique comme métaphore», art. cité, p. 6.

194. Michel Bélanger, "Considérations sur la "constitutionnalisation internationale"...», art. cité, p. 48.

195. Voir "Conflits entre normes de jus cogens", dans Droit du pouvoir, pouvoir $d u$ droit. Mélanges offerts à Jean Salomon, Bruxelles, Bruylant, 2007, p. 489.

196. L'expression se réfère à une obligation, un devoir. Voir en ce sens René Capitant, L'impératif juridique, Paris, Dalloz, 1928, p. 3.

197. L'auteur parle de «l'expression linguistique d'un "commandement" » (Théorie pure du droit, op. cit., p. 15).

198. Voir Des lois d'ordre public et de la dérogation aux lois, Paris, Cotillon-Pichon, 1899, p. 61. Italiques de l'auteur.

199. Voir par exemple CIJ, 9 avr. 1949, affaire du détroit de Corfou (fond), Rec. 1949, p. 22.

200. La Cour avait déjà posé ce principe en 2013. Voir CJUE (grande chambre), affaire C-399/11: Stefano Melloni c/Minesterio fiscal, 26 février 2013. 
contractantes à la convention EDH). Athanase Popov disait à ce propos que «si un État membre de l'UE entend s'appuyer sur la convention $\mathrm{EDH}$ pour accorder un niveau de protection des droits fondamentaux plus élevé que celui conféré par la Charte, le problème ne doit-il pas être résolu uniquement dans le cadre de l'UE ${ }^{201}$ ? » Le schéma serait constitué d'un but et des moyens. Le premier suit la norme fondamentale (promotion des droits fondamentaux) et les seconds constituent l'ensemble des règles qui l'entretiennent.

Et si la norme fondamentale est le principe fondateur de l'ordre, les règles ou normes - au sens de l'impératif kantien ${ }^{202}$ - qui la matérialisent ne sauraient, en bonne logique, se rencontrer. Une norme constitutionnelle poursuivant la réalisation de la séparation des pouvoirs pourraitelle s'opposer à une seconde norme de même nature concourant à la garantie des droits? La séparation des pouvoirs et la garantie des droits ne s'opposent-elles pas à l'arbitraire ${ }^{203}$ ? N'obligent-elles pas, toutes deux, les différents acteurs du système à un comportement mesuré? C'est dans ce sens que se justifierait une théorie juridique des contraintes. Il y aurait ainsi, en droit, des interprétations justes ${ }^{204}$. On le verra, du reste, il y a au sein de chaque ordre juridique - précisément dans les rapports entre les acteurs - un système régulateur, disons autocorrecteur, qui vise la cohésion de l'enceinte et promeut la vision entretenue par la norme fondamentale. C'est de cette considération que naîtrait l'idée d'une hiérarchie qui présente l'ordre juridique sous la figure d'une pyramide. Une "pyramide renversée» ou, comme l’a précisé Rolando Quadri, une pyramide qui «ne peut pas rester debout ${ }^{205} »$. Une pyramide

201. Athanase Popov, "L'avis $2 / 13$ de la CJUE complique l'adhésion de l'union européenne à la CEDH. Cour de justice de l'union européenne (art. $6 \$ 2$ TUE et protocole $\mathrm{n}^{\circ} 8$ au TUE)», Revue des droits de l'homme, 2015, p. 8 et 5, en ligne: http://revdh.revues.org/1065.

202. Pour l'auteur, l'impératif « ordonne immédiatement une certaine conduite qu'une autre fin que cette conduite permettrait d'atteindre». Voir Fondements de la métaphysique des mours, H. Lachelier (trad.), Paris, Hachette, 1915, p. 45-46.

203. Montesquieu, De l'esprit des lois, P. Janet (éd.), Paris, Delagrave, 1887 , livre XI, chap. VI, p. 237 : «Tout serait perdu si le même homme, ou le même corps des principaux, ou des nobles, ou du peuple, exerçait ces trois pouvoirs: celui de faire les lois, celui d'exécuter les résolutions publiques, et celui de juger les crimes ou les différents des particuliers [...]. Il n'y a point [...] de liberté si la puissance de juger n'est pas séparée de la puissance législative et exécutrice. »

204. Michel Rosenfeld, Les interprétations justes, Paris, LGDJ, 2000.

205. Voir «Le fondement du caractère obligatoire du droit international public», RCADI $1952, n^{\circ} 80$, p. 616. 
horizontale ${ }^{206}$ parce que renvoyant au même degré juridique (la norme fondamentale ${ }^{207}$ ) toutes les normes produites à partir des différentes interprétations ${ }^{208}$. Cette pyramide serait en toute hypothèse non pas déterminée a priori par un pouvoir constituant formel, mais déduite a posteriori du rapport de force qui oppose les règles de nature différente ${ }^{209}$. Le dire ainsi élimine quelque peu l'idée de concurrence et de correction qui structure le système d'énonciation du droit constitutionnel.

Or, dans notre cadre d'étude même, les normes sont produites par une série d'acteurs relevant d'ordres juridiques «opposés». Placer ces différents acteurs sur le même plan hiérarchique revient à penser un système censé s'annuler sous le coup de l'indifférence justifiée par l'indépendance des uns par rapport aux autres. Il y a donc nécessairement une hiérarchie dans la production des normes. La pratique voudrait que l'épuisement des recours internes soit suppléé par l'appel au juge communautaire ou à une instance internationale (Comité des droits de l'homme de l'ONU par exemple). C'est le principe de la subsidiarité qui marque à la fois l'autonomie des acteurs et le fondement d'une hiérarchie dans le système supranational d'énonciation du droit. Et comme on le verra, cette pratique, sans l'avoir prévu, place le juge national en position d'infériorité dans une pyramide qu'on hésite à concevoir ${ }^{210}$, mais qui n'est pas sans conséquence pour les acteurs.

206. Certains ont développé l'idée d'une pyramide au réseau. Voir François Ost et al., "De la pyramide au réseau? Vers un nouveau mode de production du droit?", Revue interdisciplinaire d'études juridiques, $\mathrm{n}^{\circ} 44$, 2000, p. 1-92.

207. Hans Kelsen disait lui-même qu'«il devient possible que la Constitution se concrétise en des formes juridique autres » (« La garantie juridictionnelle de la Constitution...», art. cité, p. 206).

208. Michel Troper, «Le problème de l'interprétation et la théorie de la supralégalité constitutionnelle», Mélanges offerts à Charles Eisenmann, Paris, Cujas, 1977, p. 135 .

209. Éric Millard, "Qu'est-ce qu'une norme juridique?», art. cité., p. 91. Certains estiment néanmoins que «la Constitution s'analyse avant tout comme une "norme de production de normes” ». Voir Ferdinand Mélin-Soucramanien, «La constitution, le juge et le "droit venu d'ailleurs" ", dans Démocratie et liberté, op. cit., p. 178. D’autres encore que la «hiérarchie peut être expressément établie par un texte fondamental ou bien découler d'une reconnaissance opérée par une autorité indépendante des auteurs des normes en cause, laquelle est chargée d'en assurer le contrôle» (Fabrice Picod, "La normativité du droit communautaire», Cah. Cons. const. 2006, $\mathrm{n}^{\mathrm{o}}$ 21, p. 145).

210. L'idée d'une hiérarchie entre les ordres nationaux et communautaires en matière de droits fondamentaux a cependant été entreprise par Louis Favoreu. Voir «Le contrôle de constitutionnalité du traité de Maastricht et le développement du "droit constitutionnel international" ", art. cité, p. 62. 


\subsubsection{Norme, contrainte, obligation}

Vue d'ensemble, la norme fondamentale astreint le système ou les différents systèmes qui en font la promotion au respect des idéaux qu'elle poursuit. Toute chose qui exige un système d'obligations et de contraintes. La contrainte se caractérise dans ce sens par une « obligation à...», mais aussi par «l'obligation de... ${ }^{211} »$.

La théorie des contraintes juridiques, qu'on pourrait convoquer ici, aurait été formulée pour moduler les effets d'une théorie réaliste de l'interprétation qui laisserait une importante marge d'appréciation aux acteurs dans le système d'énonciation du droit ${ }^{212}$. Elle est, au même titre que sa devancière (la théorie réaliste de l'interprétation), vivement critiquée $^{213}$. Olivier Jouanjan considère la seconde comme l'«adjuvant théorique » de la première. Il estime que «la théorie des contraintes vient réduire, comme à sa plus simple expression, la liberté de l'interprète que proclame la théorie réaliste ${ }^{214} »$. Michel Troper, le plus souvent visé par les critiques de l'école française ${ }^{215}$, semble avoir placé des garde-fous pour la survivance de son ingénierie.

De manière générale, il «entend par "contraintes" l'ensemble des données juridiques dont le juge constitutionnel doit tenir compte». «Il ne s'agit ici, bien entendu, que de facteurs proprement juridiques, c'est-à-dire qui tiennent à l'organisation constitutionnelle générale ${ }^{216}$.» Il disait à une date relativement reculée que l'interprétation mettait en relation les pouvoirs publics: «Dans aucun système, l'organe de contrôle de constitutionnalité n'est seul interprète de la constitution. L'interprétation qu'il donne doit donc tenir compte de celle des autres organes $^{217}$ ». C'est, semble-t-il, à partir de cette corrélation - qui participe de l'exigence de mutualité propre au système démocratique ou

211. Michel Troper, Le droit et la nécessité, op. cit., p. 8.

212. Jean-Claude Le Coustumer, "Troper Michel», dans Olivier Cayla et al. (dir.), Dictionnaire des grandes cuvres juridiques, Paris, Dalloz, 2008, p. 583.

213. Otto Pfsermann, «Contre le néo-réalisme juridique. Pour un débat sur l'interprétation », RFD comp. 2002, $\mathrm{n}^{\circ}$ 50, p. 279-334 ou encore « Une théorie sans objet, une dogmatique sans théorie. En réponse à Michel Troper », RFD comp. 2004, $\mathrm{n}^{\circ} 52$, p. $759-788$.

214. Voir "La théorie des contraintes juridiques de l'argumentation et ses contraintes", Droits, $\mathrm{n}^{\circ}$ 54, 2011, p. 27-48 (spécifiquement p. 33).

215. Nous renverrons à la critique de Stéphane Rials, «La démolition inachevée. Michel Troper, l'interprétation, le sujet et la survie des cadres intellectuels du positivisme néoclassique ", Droits, $\mathrm{n}^{\circ} 37,2003$, p. 49-85.

216. Michel Troper, Pour une théorie juridique de l'État, op. cit., p. 343.

217. "Le problème de l'interprétation et la théorie de la supralégalité constitutionnelle ", dans Mélanges offerts à Charles Eisenmann, op. cit., p. 148-150. 
constitutionnel - que l'auteur bâtit sa théorie des contraintes. Il faut à ce sujet faire deux remarques avant d'illustrer la façon dont se manifestent les contraintes dans le cadre constitutionnel qui nous intéresse.

D'une part, l'auteur reste dans la perspective du positivisme juridique en ce sens qu'il ne se réfère qu'à la contrainte exercée dans le cadre de l'organisation constitutionnelle. Il se distingue, par ce rapport (à l'organisation constitutionnelle), de l'école scandinave du réalisme juridique sous la plume des auteurs comme Olivecrona ou Ross qui considèrent l'ordre juridique non comme un système global, mais comme une «machinerie juridique» composée d'éléments divers en attente de concrétisation ${ }^{218}$. D'autre part donc, autant la manifestation de la contrainte est imprévisible dans ce cadre épars, autant elle doit l'être dans le cadre centralisé qu'entretient Michel Troper. Un cadre déduit non du rapport entre les différents énoncés textuels, mais à partir de la performance des acteurs du système. La théorie des contraintes fait donc partie intégrante du réalisme juridique sans nécessairement en constituer «l'adjuvant » puisque l'une et l'autre surpassent le normativisme classique - marqué par la référence au texte - en proposant une lecture du droit tirée des «leçons de l'expérience ${ }^{219} »$. La contrainte juridique serait dans ce cas le produit d'une interprétation authentique (au sens d'absolue) invitant les acteurs du système, autorités inférieures de fait, à suivre une certaine démarche plutôt qu'une autre. Elle est renforcée par la contrainte psychologique, la psychose de la cassation, la peur de se voir désavouer ${ }^{220}$. Le système transversal, mis en place par la constitutionnalisation institutionnelle internationale ${ }^{221}$, illustre nettement ce double rapport qui pèse sur les acteurs juridiques.

Dans ce cadre, en empruntant à Michel Troper la formule de la contrainte juridique faible qui consiste en une organisation normative des rapports affectant les «autorités de telle manière que le pouvoir des uns dissuade les autres d'exercer leur propre pouvoir discrétionnaire de façon excessive ${ }^{222}$ », on parviendrait à isoler un ensemble d'obligations

218. Éric Millard, «Le réalisme scandinave et la théorie des contraintes», communication au colloque sur Les Contraintes juridiques, Centre de théorie du droit, Université Paris X Nanterre, octobre 2000, copie de l'auteur, p. 6.

219. Voir Élizabeth Zoller, «Le réalisme dans la jurisprudence constitutionnelle de la Cour suprême des États-Unis », Cah. Cons. const. 2007, nº 22, p. 216.

220. Ce type constituerait une contrainte stratégique selon Michel Troper. Voir "Argumentation et explication", Droits, $\mathrm{n}^{\circ}$ 54, 2011, p. 25.

221. Voir Michel Bélanger, "Considérations sur la "constitutionnalisation internationale"...", art. cité, p. 49-50.

222. Voir La théorie du droit, le droit, l'État, op. cit., p. 95. 
entretenant l'idée de contraintes juridiques. Le juge communautaire - dans le cadre européen - a par exemple tracé certaines bornes à l'adresse des autorités communautaires et étatiques. Miguel Herrero de Miñón fait remarquer que sur un demi-siècle d'activité, la CJUE a posé pas moins de quatre techniques d'interprétation des textes communautaires: "L'effet utile", affirmé en 1957 (cas Algera), a été substitué par l'interprétation téléologique (cas Van Gend en Loos, 1963, Costa c/Enel 196, et avis 1/1992), l'effet nécessaire (cas ATR, 1971) et l'interprétation globalisante». Ces modes de raisonnement juridiques constituent bien des contraintes, c'est-à-dire des procédés de validité des normes qui obligent les autorités publiques à un comportement précis. L'auteur y voit d'ailleurs un acquis communautaire " des caractéristiques constitutionnelles $^{223}$ ». L'usage de l'une de ces clauses interprétatives dans l'examen du projet d'accord portant adhésion de l'UE à la convention EDH aurait sans doute produit un avis 2/13 différent ${ }^{224}$. L'affaire Van Gend en Loos est aussi régulièrement citée comme fondatrice d'une obligation qui pèse sur le juge national. Il en va de même de la jurisprudence Costa du 15 juillet 1964 qui affirme «l'impossibilité pour les États de faire prévaloir, contre un ordre juridique accepté par eux sur une base de réciprocité, une mesure unilatérale ultérieure qui ne saurait ainsi lui être opposable ${ }^{225} »$. Ferdinand Mélin-Soucramanien en parle en termes d'arrêt fondateur d'une dynamique d'intégration juridique ${ }^{226}$. Fabrice Picod fait, à la suite, remarquer que «le droit communautaire crée des obligations à l'égard des États, mais aussi des particuliers, ce qui lui confère un attribut fondamental de la normativité». L'auteur précise que la hiérarchie entre les deux ordres est protégée par un «système de contrôle qui permet d'en sanctionner les violations ${ }^{227}$ ». Le précédent auteur conclurait de là que «le principe de primauté revêt une portée absolue et le droit communautaire "brise" le droit national, y compris

223. Miguel Herrero de Miñón, «Pour le peuple mais sans le peuple. Parallélismes entre la Constitution européenne et le constitutionnalisme philo-soviétique», dans La constitution et les valeurs, op. cit., p. 412.

224. Un auteur fait remarquer que la CJUE a usé d'une méthode d'interprétation littérale plutôt que juridique. Voir Athanase Popov, "L'avis 2/13 de la CJUE complique l'adhésion de l'union européenne à la CEDH... ", art. cité, p. 5.

225. Fabrice Picod, «La normativité du droit communautaire», op. cit., p. 143 et suiv. et ici p. 147.

226. Voir "La constitution, le juge et le "droit venu d'ailleurs" ", dans Démocratie et liberté, op. cit., p. 177.

227. Fabrice Picod, «La normativité du droit communautaire», op. cit., p. 142. Nous soulignons. 
constitutionnel $^{228} »$. Il pèse dès lors sur les autorités des États membres une double contrainte juridique et psychologique ${ }^{229}$.

On peut aussi citer, dans le cadre universel, la cristallisation par la Cour internationale de justice $\left(\mathrm{CI}^{230}\right)$ en standards juridiques du contenu matériel de la norme impérative qui, aux termes de l'article 53 de la convention de Vienne de 1969 relative au droit des traités, obligent les États et l'ensemble des sujets du droit international. Michel Bélanger inscrit dans le même contexte l'interprétation de la notion de paix par l'assemblée générale et le Conseil de sécurité des Nations unies. Pour l'auteur, les résolutions 33/73 du 15 décembre 1978 portant déclaration sur la préparation des sociétés à vivre dans la paix, 39/11 du 8 novembre 1984 portant déclaration sur le droit des peuples à la paix ou encore 1325 du 31 octobre 2000 relative aux droits des femmes à la paix internationale participent du mouvement de constitutionnalisation normative internationale ${ }^{231}$. La pratique transversale du droit est donc fortement marquée par des obligations et contraintes qui lient les autorités publiques. Le juge national n'a d'ailleurs pas souvent hésité à appliquer une norme supranationale au détriment d'une norme de droit interne. On en trouve un exemple dans l'affaire UNDP c/État du Cameroun $^{232}$ dans laquelle le juge camerounais convoque l'article 21 de la déclaration de 1948 (au détriment des textes nationaux) pour constater la fraude électorale fondée, entre autres, sur l'intimidation des scrutateurs $^{233}$.

La contrainte juridique se trouve être, dans ce cadre, la condition d'existence de l'ordre juridique supra-étatique ${ }^{234}$. La contrainte revêt les attributs juridiques parce que le cadre matériel et procédural de la vie juridique est clairement tracé par l'interprétation des textes fondateurs. Elle est tout aussi psychologique du fait que les autorités étatiques sont

228. Voir «La constitution, le juge et le "droit venu d'ailleurs" ", op. cit., p. 177.

229. Celle-ci proviendrait, suivant Michel Troper, de la conscience de l'existence d'une norme supranationale qui serait déterminante pour les acteurs nationaux. Voir Le droit et la nécessité, op. cit., p. 11.

230. CIJ, 9 avr. 1949, affaire du détroit de Corfou (fond), Rec. 1949, p. 22 ou 5 fév. 1970, affaire Barcelona Traction (fond), Rec. 1970, p. 32.

231. Voir "Considérations sur la "constitutionnalisation internationale"...», op. cit., p. 46-48.

232. Cour suprême, 3 juin 1997, arrêt nº 22/C.E./96-97.

233. Eyike-Vieux, «Le droit international devant le juge camerounais: regards d'un magistrat», Juridis Périodique, $\mathrm{n}^{0}$ 63, 2005, p. 102.

234. Denis Alland disait: «le droit communautaire est supérieur au droit interne ou n'est pas» («Le droit international "sous" la Constitution de la $\mathrm{V}^{\mathrm{e}}$ République», RDP 1998, nº 5-6, p. 1654. 
conscientes que le moindre écart, la moindre irrégularité engage la responsabilité de l'État et entache la notoriété de l'auteur interpellé. Les juridictions nationales s'attachent désormais à cette logique, car elles rentrent dans une vaste cité institutionnelle dérivée de l'expression «cité constitutionnelle» entretenue par Dominique Rousseau ${ }^{235}$.

\subsection{Une unité institutionnelle}

Ainsi qu'il apparaît des précédents développements, l'organisation institutionnelle au-delà du cadre étatique fonctionne comme dans un contexte centralisé. On observe que l'UE par exemple jouit d'une double personnalité juridique interne et internationale avec un parlement législateur au sens complet du terme et un conseil qui, à l'instar d'un pouvoir exécutif national, peut internationalement engager l'Union ${ }^{236}$. Il y a, toute proportion gardée, un fonctionnement institutionnel bien établi qui oblige le chercheur à constater une organisation constitutionnelle. Mais du cadre étatique au cadre supra-étatique, l'organisation constitutionnelle impose une exigence terminologique. Elle pourrait se résumer dans cette étude par la question de savoir si la notion d'institution régulièrement évoquée traduit une spécificité ou un choix lexical. Y aurait-il une différence fondamentale entre institution et organe? Les institutions évoquées jouissent-elles d'un véritable pouvoir? Voilà au moins deux préoccupations qui ne laisseront pas le juriste indifférent (2.2.1). Une autre interrogation, certainement pas la dernière, se rapporte aux différents rapports qui naîtraient entre les acteurs dans ce cadre (2.2.2).

\subsubsection{Institutions ou organes?}

Le recours à la notion d'institution dans l'analyse du phénomène juridique au-delà du cadre national ${ }^{237}$ traduit-il une simple fantaisie? Cache-t-il, au contraire, un impératif méthodologique, une exigence du vocabulaire juridique? L'usage des concepts d'organe ou de représentants aurait-il produit des conséquences autres? Ainsi se décline le projet des lignes qui vont immédiatement suivre.

235. Voir «Le droit constitutionnel en débat, le droit constitutionnel européen existet-il ?», RDP 2008, n 3, p. 725.

236. Jérôme Roux, Droit général de l'union européenne, op. cit., p. 175 et suiv et p. 193 et suiv.

237. Emmanuel Decaux, dans le cadre du droit international, oppose le droit relationnel au droit institutionnel. Voir «Déclarations et conventions en droit international», Cah. Cons. const. 2006, $\mathrm{n}^{\mathrm{o}} 21$, p. 138. 
La notion d'institution caractérise le relief juridique d'une organisation sociale. Elle constitue, chez Maurice Hauriou, une opération par et pour laquelle un groupe s'organise et parvient à une communion politique $^{238}$. Il se dégage de son étude un double axiome du reste complémentaire. D'une part, explique Éric Millard, l'institution est « un concept essentiel de l'analyse». En ce sens, son évocation offre une perspective autre, différente de celle du contrat par exemple, dans l'appréhension du fondement des sociétés politiques. D'autre part donc, l'institution est «le processus qui institue un groupe humain». Et à l'auteur d'insister, «moins que la forme instituée, elle [l'institution] est la constitution de cette forme dans la durée». Il suit que l'institution n'est pas seulement l'objet d'analyse du phénomène politique, elle en est aussi l'«outil immédiat ${ }^{239} »$. Cette acception pourrait avoir séduit les auteurs.

La perspective est d'autant plus plausible que l'institution a le mérite de résoudre l'équation de l'éthos qui se pose au-delà du cadre national ${ }^{240}$. L'analyse institutionnelle présente donc, à la fois, les aspects politique et juridique de l'organisation sociale. Elle permet d'une part de présenter la société en corporative, une organisation sociale objective ayant pour vocation de réaliser «l'état de droit le plus haut ${ }^{241}$ ". La référence à l'institution permettrait dans cette perspective de dépasser l'axiologie classique d'un droit supra-étatique essentiellement relationnel puisque, précisément, l'institution s'inscrit en réaction à l'extension abusive du contrat $^{242}$. L'usage du concept d'institution caractérise ainsi la postmodernité du droit dans le cadre auquel on se rapporte. Elle permettrait, dirait Anne Peters, d'assurer la stabilité légale et « la prévisibilité » qui font généralement défaut à l'ordre international ${ }^{243}$. D'autre part, l'institution traduit l'idée de légitimité dont la performance se mesure à l'adhésion de la société civile à tout projet juridique. Éric Millard y voit un instrument d'évaluation de la réaction subjective à une conception objective de la société: la manifestation de communion, «l'adhésion de chacun des

238. «La théorie de l'institution et de la fondation. Essai de vitalisme social», dans Aux sources du droit: le pouvoir, l'ordre et la liberté, Cahiers de la Nouvelle Journée, $\mathrm{n}^{\circ}$ 23, 1990, cité par Éric Millard, «Hauriou et la théorie de l'institution », Droit et sociétés, $\mathrm{n}^{\circ} 30 / 31,1995, \mathrm{p} .392$.

239. Ibid., p. 385.

240. François Borella, «La situation actuelle du droit constitutionnel», art. cité, p. 4-7.

241. Voir Maurice Hauriou, Principes de droit public, Paris, Sirey, 1916, p. 111.

242. "C'est en réaction contre [l'] extension abusive du contrat qu'est née la théorie de l'institution ", disait Jean-Louis Bergel (Théorie générale du droit, Paris, Dalloz, 1989, p. 184).

243. Voir « Reconstruction constitutionnaliste du droit international... », op. cit., p. 371. 
membres ${ }^{244}$ ». L'auteur rejoint Alf Ross dans l'exigence des «protocoles de vérification ${ }^{245}$ » qui se résume, non pas dans une démocratie directe sur le modèle cantonal suisse, mais, dans la fonction institutionnaliste de la filiation à l'ordre juridique. On revient au système d'imputation dont l'idée est toute faite à l'attribut juridique des «commandements énoncés par des êtres humains spécialement investis de ce pouvoir dans le but de diriger la conduite d'autres êtres humains ${ }^{246}$ ». Dès lors, la notion d'institution rencontre celle de l'ordre juridique, suivant l'unité développée par Kelsen. Elle traduit, dirait Pierre Brunet, «la personnification de toutes les actions juridiquement déterminées, c'est-à-dire de toutes les actions réglées par l'ordre juridique ${ }^{247}$ ». Le recours à la notion d'institution ne dispense donc pas l'ordre juridique supranational des analyses liées à la connaissance juridique de l'État. Ce faisant, la notion d'institution pourrait indifféremment valoir celle d'organe. Cela nécessite un inventaire.

Si l'institution traduit en termes juridiques le fondement de l'unité politique ou sociale tout en se substituant à cette unité, peut-elle être assimilée à l'organe? Tout semble l'indiquer. D'abord l'organe, comme l'institution, permet, suivant Oscar Orban, de justifier l'idée de personnification de la société (l'éthos) par l'ordre juridique ${ }^{248}$. L'image de l'organisme, développée par Gerber, est en effet le résultat du rapprochement de la société et de la personne juridique ou de l'État. Franco Fardella dira également que "la métaphore organique ne semble pas être dénuée d'implications métaphysiques dérivées du vitalisme ${ }^{249}$ ». Ensuite donc, l'organe justifie «l'attribution » de la volonté, disons de la personnalité, à l'ordre juridique. Pierre Brunet précise dans cette veine que «sans organes, l'État [ou l'ordre juridique] n'a pas de volonté ${ }^{250}$ ». Il remplit lui aussi la fonction d'imputation. On retrouve cette idée chez Jellinek, Kelsen ou encore Carré de Malberg. Elle se résume à ceci : est organe, tout individu dont les actes sont considérés comme ayant été

244. Voir « Hauriou et la théorie de l'institution», op. cit., p. 394-397.

245. Expression empruntée à Éric Millard, Une théorie empirique du droit est-elle possible? Lectures d'Alf Ross, Droit et Société, $\mathrm{n}^{\circ}$ 50, 2002, p. 15.

246. Véronique Champeil-Desplats, «Alf Ross : droit et logique», art. cité, p. 31.

247. Voir «Alf Ross et la conception référentielle de la signification en droit», Droit et Société, $\mathrm{n}^{\circ} 50,2002$, p. 25.

248. Voir Oscar Orban, Le droit constitutionnel de la Belgique, Liège, Nabu Press, 2010, p. 459 et suiv.

249. Cité par Franco Fardella, «Le dogme de la souveraineté de l'État: un bilan », Arch. phil. dr. 1997, nº 41, p. 120 et voir aussi p. 129.

250. Voir Pierre Brunet, Vouloir pour la nation. Le concept de représentation dans la théorie de l'État, Paris, LGDJ, 2004, p. 48. 
pris au nom de l'ordre juridique. On dira que ces actes sont imputables à cet ordre juridique et non à l'être de chair et de sang qui les a pris. Georg Jellinek disait d'ailleurs: "Derrière le représentant, il y a une personne; derrière l'organe, il n'y a rien ${ }^{251}$.» Dès lors, la CEDH, qu'on connaît sous l'étiquette d'institution, ne serait pas moins un organe du moment où les actes qu'elle prend sont imputés à l'ordre juridique européen. Le critère de qualification de compétences développé par Alf Ross dans la signification des organes de l'État contribue elle-même à cette conclusion ${ }^{252}$. Organes et institutions permettraient ainsi de séparer l'ordre politique de l'ordre juridique. Pourraient-ils, ce faisant, justifier les attributs de représentants ou la notion de représentation au-delà du cadre national ?

Il paraît aujourd'hui courant d'utiliser indifféremment les concepts d'organes et de représentants. L'un comme l'autre réalise l'unification, au moyen de la technique juridique, des antagonismes sociologiques de la société politique. L'un comme l'autre décrit l'écart entre la société civile et l'ordre juridique. On pourrait donc dire que même derrière le représentant il n'y a rien. La représentation en théorie du droit déroge en effet aux règles du Code civil. Il s'agit d'un mandat de droit public $^{253}$ qui justifie la formulation de la volonté générale ${ }^{254}$. Ce n'est pas tant le mandat qui détermine le statut du représentant que sa fonction : est représentant celui qui participe à la formation de la volonté générale $^{255}$. Éric Maulin conclura pour sa part à «l'impossible présence du représenté dans le champ de l'organisation politique ${ }^{256} »$. L'idée est celle d'une représentation indifférenciée constitutionnellement traduite par le principe de la nullité du mandat impératif ${ }^{257}$. Le représentant et l'organe renvoient donc usuellement à la même réalité. Carré de Malberg estime que «la Constituante a entendu le mot de représentation dans

251. Cité par Léon Duguit, L'État le droit objectif et la loi positive, Paris, Dalloz, 2003, p. 9.

252. Voir «Sur les concepts d'“État" d'“organes d'État" en droit constitutionnel», Droits, $\mathrm{n}^{\circ} 2,1996$, p. 144.

253. L'idée est développée par l'école de l'Isolierung qui considère l'organe comme une représentation institutionnelle. Voir Olivier Beaud, « La souveraineté dans la "Contribution à la théorie générale de l'État" de Carré de Malberg ", RDP 1994, $\mathrm{n}^{\mathrm{o}} 1, \mathrm{p} .1281$.

254. Philippe Blachèr, Contrôle de constitutionnalité et volonté générale, op. cit., p. 175.

255. Pierre Brunet, «La notion de représentation sous la Révolution française », $A H R F$, $\mathrm{n}^{\mathrm{o}} 2,2002, \mathrm{p} .30$.

256. Voir «Démocratie et représentation dans la pensée de Carré de Malberg», Droits, $\mathrm{n}^{\mathrm{o}} 22,1995$, p. 127.

257. Art. 15-3 de la Constitution du Cameroun. 
le sens même où s'emploie aujourd'hui celui d'organe: dans le régime institué par elle, ce mot signifiait que les prétendus représentants sont, non pas les interprètes d'une volonté nationale qui puisse se former en dehors d'eux, mais les organes par lesquels se forme cette volonté 258 ».

Mais évoquer la représentation au-delà du cadre national revient à s'attaquer à un problème précis : la loi, expression de la volonté générale. On a en effet relevé que c'est de la formation de la volonté générale que se déduit le statut de représentant. Or la loi dont on parle est exclusivement l'acte du Parlement (dixit le Conseil constitutionnel français, 85-197 DC, 23 août 1985: «la loi votée»), un corps typique de l'État. Les actes des parlements communautaires n'ayant pas encore revêtu la qualification de «loi» au sens formel (ce qui est curieux), il est à se demander si les eurodéputés par exemple sont les représentants des différentes nations qui peuplent l'Union. Admettre une telle hypothèse reviendrait à déplacer les usages du mot et à revenir donc au statut posé par le Code civil: une relation entre représentant et représenté. Cette thèse est de plus en plus évoquée. Stéphane Pierré-Caps disait dans ce sens que la représentation ne traduit plus seulement «l'indifférenciation du peuple souverain dans l'État», mais la présentation « d'un élément spécificateur (religion, ethnie, localité, sexe) auquel on se doit d'être fidèle dans l'exercice de la fonction ${ }^{259}$ ». Qu'on la réfute dans le discours juridique n’y change rien. Les sensibilités (écologistes, socialistes...) développées au sein des parlements communautaires la justifient. La réfuter revient d'ailleurs à faire de la notion de parlement, au dos de l'arrêt Matthews $c / R U$ de 1999 par lequel la CEDH appelle le Parlement européen un "corps législatif», une simple nominalisation ${ }^{260}$. La volonté générale requise dans les discours devient elle aussi irréalisable au-delà du cadre national, sauf à prendre la loi dans son sens matériel, c'est-à-dire un acte juridique impersonnel et de portée générale dont le sens pourrait être attribué aux directives communautaires par exemple. Quoi qu'il en soit, et quel que soit le nom qu'on donne aux instances supraétatiques, l'ordre juridique est perceptible au-delà du cadre national et les influences de ces instances se ressentent au sein des États.

258. Voir «Le droit constitutionnel entre universalisme et particularisme», art. cité, p. 298.

259. Voir «Les minorités et la représentation», Cah. Cons. const. 2007, $\mathrm{n}^{\circ} 23, \mathrm{p} .87$.

260. Voir pour le vocable nominalisation des concepts Jean Combacau, «Pas une puissance, une liberté : la souveraineté internationale de l'État», Pouvoirs, n ${ }^{\circ} 67$, 1993, p. 48. 


\subsubsection{Hiérarchie entre institutions ou administration institutionnelle au réseau?}

On remarque, à partir des logiques de fonctionnement des institutions judicaires tout au moins, un dédoublement d'organes nationaux et supranationaux qui appelle la conclusion d'un ordre juridictionnel structuré en paliers.

On a d'une part le renvoi préjudiciel qui place les juridictions nationales et communautaires dans une dynamique de réseau ${ }^{261}$. Le principe consiste pour une cour, inférieure, mais plus nettement incompétente, de surseoir jusqu'au règlement de la question de la compétence en jeu. L'exemple français depuis l'avènement de la question prioritaire de constitutionnalité est éloquent. On part d'une juridiction d'instance qui transmet la question à la cour supérieure de l'ordre juridictionnel auquel elle appartient; celle-ci renvoie la question au Conseil constitutionnel à qui revient la compétence de statuer sur l'hypothèse d'inconstitutionnalité $^{262}$. Il y a là un système de coopération constitutionnellement établi.

C'est en des termes quasi identiques que fonctionne le renvoi préjudiciel au-delà du cadre national. On parle d'une «collaboration étroite et originale [...] efficace pour le juge de l'Union afin de consacrer certains principes structurants du processus d'intégration ${ }^{263}$ ». Dans le contexte africain, on pourrait évoquer la pratique au sein de la CEMAC. À ce titre, l'article 17 de la convention régissant la CJCEMAC dispose que la chambre judiciaire statue à titre préjudiciel sur les questions ayant trait à l'interprétation des textes fondateurs et sur la légalité de l'institution en général dès lors qu'une juridiction nationale " est appelée à en connaître à l'occasion d'un litige». Le texte précise que la compétence de la chambre est absolue; que, lorsqu'une juridiction nationale, saisie des questions de légalité, doit se prononcer en dernier ressort, le renvoi devant la chambre demeure un préalable. Cette saisine préalable de l'institution communautaire a pour fonction d'assurer la prééminence du droit communautaire et, par voie de fait, celle du juge qui en est le garant. Il est précisé à ce propos aux termes de l'article 18 de la convention suscitée que les décisions en interprétation de la légalité prises par la chambre judiciaire s'imposent aux autorités administratives et juridictionnelles au sein des États membres. Cette idée de primauté ne semble pourtant pas totalement admise. Ainsi, pour certains, le renvoi

261. Mireille Delmas-Marty, Les forces imaginantes du droit, op. cit., t. III, p. 83-89.

262. Guy Carcassonne et al., QPC La question prioritaire de constitutionnalité, Paris, Dalloz, 2012, p. 39-59.

263. Chahira Boutayeb, Droit institutionnel de l'union européenne, op. cit., p. 388. 
place les autorités nationales et supranationales non pas dans un rapport hiérarchique, mais au sein d'un système de coopération, c'est-à-dire une organisation structurée. Ce faisant, le renvoi promeut une «unité de droit».

D'autre part, on a les recours directs et la procédure de subsidiarité très performants en matière de protection des droits et libertés fondamentaux. Premièrement, la procédure de contrôle direct a " pour objet la constatation des manquements éventuels commis par les États membres à leurs obligations». Il s'agit d'un type de procédure particulier qui s'apparente à la fois au contentieux de droit commun et à un contentieux administratif $^{264}$. C'est dans ce sens qu'on lira l'article 14 de la convention instituant la CJCEMAC ${ }^{265}$. Jacques Pertek écrit, à partir du contexte européen, que ces recours concourent à la protection des particuliers dont les plaintes «sont devenues le principal moyen pour la commission de détecter les manquements des États membres ${ }^{266}$ ». Deuxièmement, le recours en manquement, défini à partir de l'article 4 du traité révisé de la CEMAC comme le fait pour un État de s'abstenir de prendre des mesures concourant à la promotion des objectifs communautaires ou de prendre des mesures qui pourraient faire obstacle à la réalisation de telles mesures, est perceptible à partir de la technique de subsidiarité. Il est très usité en cas de violations des droits fondamentaux. La subsidiarité est alors regardée comme une technique performante en matière de protection des droits de l'homme. Elle signifie que la saisine de la cour communautaire est subordonnée à l'épuisement des voies de recours internes. Ce système illustre ainsi l'unité institutionnelle et le dédoublement organique. Denys Simon y verrait « une structure constitutionnelle qui se reflète dans le principe des compétences d'attribution ${ }^{267} »$. Cette gouvernance institutionnelle des droits fondamentaux illustre dans tous les cas l'idée d'une constitution au-delà du cadre étatique. Elle s'entend chez Pavle Nikolic comme une problématique de l'État de droit: celui-ci désigne «non seulement l'existence d'une Constitution, comme expression et garantie suprême des droits et libertés de l'homme et du

264. Jérôme Roux, Droit général de l'Union européenne..., op. cit., p. 325, 383, 351 et suiv.

265. Le texte reconnaît la qualité de requérant à toute personne qui justifie d'une violation « des dispositions des Traités de la C.E.M.A.C et des Conventions subséquentes » tout en lui reconnaissant le droit de soulever, lors d'un procès, l'exception d'illégalité d'un acte juridique d'un État ou d'un organe communautaire.

266. Jérôme Roux, Droit des institutions de l'Union européenne..., op. cit., p. 399.

267. «Deuxième (ou second et dernier?) coup d'arrêt à l'adhésion de l'Union à la CEDH...», art. cité, p. 3. 
citoyen, ainsi que d'autres acquis de la civilisation et de la démocratie, mais [aussi et surtout] le principe et les garanties de cette suprématie constitutionnelle ${ }^{268}$ ». L'idée d'une «constitution internationale» situe ainsi les impératifs de la démocratie et la promotion des droits fondamentaux en général dans un vaste système juridique.

L'idée de "constitutionnalisme multiniveaux» chère à Neil Walker mérite une mise en perspective. Elle s'inscrit dans le cadre de la postmodernité du droit et requiert un déploiement théorique au rebours des cadres tracés par le constitutionnalisme des Lumières. L'auteur parle d'une approche transcendant le cadre politique et étatique ${ }^{269}$. Une question vient rapidement à l'esprit : comment concilier, au-delà du cadre national, les rapports peuple/constitution ou encore État/constitution. C'est dire combien l'idée d'une "constitution internationale» excite la curiosité. Il faut même croire qu'elle n'est pas réalisable à partir des principes consacrés par le droit constitutionnel révolutionnaire. Georges Scelle faisait le constat suivant : «Si l'on part d'une conception "formelle" du Droit constitutionnel, - de l'État - de la Société internationale, telle que nous l'offre la doctrine classique, basée sur un certain nombre de représentations ou d'idéologies familières à nos milieux sociaux nationaux, et selon nous, déformées par leur exclusivisme même, il est impossible de concevoir le sens véritable du problème ${ }^{270} »$. Il faut donc nécessairement contourner le sacré, pour ne retenir - opportunément que «l'essentiel».

$\mathrm{La}$ « constitution internationale» serait ainsi, du point de vue formel, la norme fondamentale du commerce juridique post-étatique et plus ou moins métapolitique qu'avait entrevue le philosophe Jürgen Habermas. Entendue comme telle, la "constitution internationale " correspond à un ou au mieux à l'ensemble des règles de référence (nationales et supranationales) qui concourent à la réalisation de la synthèse des différents ordres juridiques dont les divergences sont plus nombreuses que les convergences. On a parlé d'une fédération de systèmes juridiques. Il se pose une autre difficulté liée à la production des normes au sein de cette unité. En réalité la question est celle d'une possible concurrence entre les différents acteurs de ce système de plus en plus centralisé.

268. «Le contrôle juridictionnel de la constitutionnalité des lois dans le système de l'État de droit. Esquisse pour une approche globalisante», dans Démocratie et liberté, op. cit., p. 194.

269. Voir «Le constitutionnalisme multiniveaux», art. cité, p. 442.

270. «Le droit constitutionnel international», art. cité, p. 503. 
La réponse consiste à ne voir en la norme qu'un commandement qui oblige un certain comportement pour la survie de l'ordre global. Ainsi, les acteurs du système travaillent dans une dynamique de réseau, on dira de collaboration dans lequel chacun tient compte des autres avec un souci d'équilibre et de notoriété. L'idée d'une possible hiérarchie n'aurait ainsi de sens que par rapport à la performance des acteurs puisque la norme en vigueur (dans le sens de celle qui produit des effets juridiques) témoigne de la place qu'occupe son auteur dans l'ensemble du système.

On comprend que le sens matériel, qui vise la connaissance de toute cette pratique institutionnelle, ne saurait se distinguer de l'aspect formel $^{271}$. Celui-ci étant en dernière analyse lié au vœu de préserver les éléments vitaux de l'ordre juridique global que sont la promotion et la protection des droits et libertés fondamentaux. L'intérêt de l'articulation des sens formel et matériel de la constitution se retrouve dans l'impératif de la construction d'une nouvelle société plus intégrée qui réapproprierait la souveraineté (nationale) aux peuples par la pratique d'une représentation immédiate dont la saisie (directe) des instances supranationales est le gage.

La question de la corrélation entre la constitution et l'État trouverait quant à elle une solution amiable si l'on s'en tient aux prescriptions de l'école du positivisme juridique qui ne voit ni l'une ni l'autre comme une donnée naturelle. On aura alors un territoire défini comme espace de référence où se meuvent l'ordre juridique visé (Schengen en Europe en est un), un peuple déterminé par l'adhésion aux valeurs juridiquement déterminées et un gouvernement composé des instances nationales et supranationales qui contrôlent le respect des valeurs et mieux encore de la norme fondamentale (posée et construite par les différentes interprétations des acteurs du système juridique auquel on se rapporte). L'état actuel du monde oblige à ne pas ignorer les catégories juridiques au-delà du cadre étatique, cela reviendrait à s'abandonner aux conjectures politiques. Et comme l'a écrit Muriel Fabre-Magnan : « [1]a neutralité axiologique n'existe pas en droit, et le refus de choisir entre

271. En général, disait Hans Kelsen, «la Constitution au sens matériel coïncide avec la Constitution au sens formel» (« La garantie juridictionnelle de la Constitution...», art. cité, p. 205). 
plusieurs valeurs entraîne de fait souvent un choix de société à l'exclusion des autres ${ }^{272}$.»

Docteur de l'université de Dijon Membre associé du Centre de recherche et d'étude en droit et science politique (CREDESPO, université de Bourgogne)

Maître-assistant du Conseil africain et malgache pour l'enseignement supérieur (CAMES)

Chargé de cours à la faculté des sciences juridiques et politiques, université de Ngaoundéré (Cameroun) 